ESAIM: M2AN 50 (2016) 1631-1657

DOI: $10.1051 / \mathrm{m} 2 \mathrm{an} / 2016001$
ESAIM: Mathematical Modelling and Numerical Analysis

www.esaim-m2an.org

\title{
COMPARISON OF TWO-FLUID MODELS ON STEAM-WATER TRANSIENTS
}

\author{
Hippolyte Lochon $^{1,2,5}$, Frédéric Daude ${ }^{1,2}$, PASCAl Galon ${ }^{2,3}$ \\ AND JEAN-MARC HÉRARD ${ }^{4,5}$
}

\begin{abstract}
This paper is devoted to the comparison of three two-fluid models in steam-water applications involving phase transition and shock waves. The three models are presented in a common formalism that helps to underline their shared properties. A numerical method based on previous work is extended to all models and to more complex Equations Of State. Particular attention is paid to the verification of every step of the method so that convergence studies can be carried out. Afterwards, models are compared with each other and with experimental data in two different cases of steamwater transients. The first one is Simpson water-hammer experiment and the second one is a rapid depressurization with flashing studied in Canon experiment.
\end{abstract}

Mathematics Subject Classification. 76T10, 35L60, 35L67.

Received May 29, 2015. Revised November 5, 2015. Accepted January 5, 2016.

\section{INTRODUCTION}

Two distinct approaches are commonly used in order to model two-phase flows: the homogeneous approach and the two-fluid one. Homogeneous models consider the mixture as one fluid with its proper thermodynamical properties based on some hypothesis on the equilibria between phases. On the contrary, the two-fluid approach studies the evolution of two different fluids including many possible phasic desequilibria. In this paper, we focus on the second approach which is assumed to be more general.

The construction of two-fluid models is usually based on an averaging procedure of the local conservation laws $[14,26]$. As no phasic equilibrium is assumed, closure laws need to be provided so that the model is fully defined. A classical approach to obtain those closure laws is to require some properties of the corresponding model. Thus, we choose to restrict the present work to models that comply with a physically relevant entropy inequality and exhibit unique jump conditions. We emphasize the fact that both properties are crucial from the numerical point of view in order to obtain grid converged solutions. Moreover, as pointed out in [5], the classical

Keywords and phrases. Two-phase compressible flows, entropy inequality, closure laws, relaxation effects, finite volume schemes.

1 EDF R\&D, Département AMA, 1 Avenue du Général de Gaulle, 92141 Clamart cedex, France. hippolyte.lochon@edf.fr

2 IMSIA, UMR EDF/CNRS/CEA/ENSTA 9219, 1 Avenue du Général de Gaulle, 92141 Clamart cedex, France.

3 CEA, DEN, DANS, DM2S, SEMT, DYN, 91191 Gif-sur-Yvette cedex, France.

4 EDF R\&D, Département MFEE, 6 Quai Watier, 78401 Chatou cedex, France.

5 I2M, UMR CNRS 7373, Technopôle Château-Gombert, 39 rue F. Joliot Curie, 13453 Marseille cedex 13, France. 
hypothesis of a pressure equilibrium leads to models that don't comply with those requirements. Therefore, we will only focus on the class of two-pressure models in this paper. Ransom and Hicks [35] have proposed one of the first models in this class. More recently, Baer and Nunziato [3] have proposed another model that belongs to this class in the frame of granular reactive materials. This model has received a lot of attention in the literature and has been applied to a wide range of applications using different closure laws [7, 19,20,28,38]. Among those different closure laws, the choice proposed by Coquel et al. [7] as well as the one from Baer and Nunziato [3] comply with the previous requirements. It leads to the three models (one for the first choice of closure laws and two for the second choice) that will be examined in this paper.

Whereas the three models share similar properties, they show different behaviors from a purely convective point of view. Those differences are even more striking in the case of vanishing phases since the closure laws from Baer and Nunziato don't depend on the void fraction. However, the models not only include convective terms but also source terms that incorporate the different relaxation phenomena between phases. Thus those source terms should reduce the gap between the three models depending on the stiffness of the relaxation process, particularly the velocity relaxation. Unlike most of the available work in the literature, we consider non-instantaneous relaxations with physical evaluations of corresponding time scales so that the consequence on the gap between the models is more intricate. Once again, we underline the fact that we don't consider an instantaneous pressure relaxation which could induce numerical instabilities [17].

Based on previous work [24], a fractional step method has been used to approximate solutions of the three models. The first step of the method is an evolution step that takes into account the convective part of the model. In the case of the Baer and Nunziato models, lots of convective solvers have been proposed $[1,2,8,12,39,41]$ unlike in the case of the model from Coquel et al. [7]. We choose to use a simple Rusanov scheme so that it could be easily applied for all models and should not interfere in the comparisons. The second step of the method is dedicated to the treatment of relaxation phenomena. The approach used in this step is similar to the one proposed in [24] for Baer and Nunziato models but the remaining model [7] requires specific work. For example, the pressure relaxation step involves a more complex scheme in this case. Extensions of the different substeps to more general equations of state (EOS) are also proposed. Particular attention is paid to the verification of each step and substep of the method. Grid convergence studies have been carried out for most of steps of the method in [24] and similar work is done here for the remaining steps of the different models.

Once the numerical methods for the three models have been verified, the comparison of those two-fluid models on steam-water transients can be tackled. Two experimental setups involving two-phase water flows with strong pressure waves have been chosen: the Simpson experiment which is a classical water hammer test case and the Canon experiment which consists in a sudden depressurization of hot liquid water. Grid convergence studies have also been carried out in those cases so that we can compare converged results of the different models with each other and with the experimental data as well.

Hence the paper is organized as follows. We first present the two-fluid models with the different closure laws and the corresponding source terms. We also recall the main properties of the models. Then the fractional step method used for the computations is presented. It includes the numerical treatment of convective terms as well as sources terms. A numerical example where we point out the differences between the convective part of the models is detailed. The last section of the paper presents the comparisons of the three models on the Simpson and Canon experiments.

\section{TWO-FLUID MODELS}

The derivation of two-fluid models used in the sequel relies on a classical two-step approach $[14,26]$. The first step is a statistical averaging of the local conservation equations which provides an open set of equations. The second step consists in closing this set of equations and the entropy inequality is a major tool to this end. Many details on this approach for seven-equation models can be found in $[25,32]$. 


\subsection{Governing equations}

Throughout the paper, subscript $k$ refers to the indexes of the phase $(k=1,2)$. Thus we denote $\left.\alpha_{k} \in\right] 0,1[$ the statistical void fraction of phase $k$, which complies with:

$$
\alpha_{1}+\alpha_{2}=1
$$

The mean density, mean velocity and mean pressure of phase $k$ are denoted $\rho_{k}>0, u_{k}$ and $p_{k}$ respectively. We also define the partial masses as:

$$
m_{k}=\alpha_{k} \rho_{k} .
$$

Moreover the specific total energy of phase $k$ is given by the following equation:

$$
e_{k}=\varepsilon_{k}+\frac{1}{2} u_{k}^{2}
$$

where $\varepsilon_{k}$ is the specific internal energy of phase $k$, linked to the density and pressure by an Equation Of State (EOS):

$$
\varepsilon_{k}=\varepsilon_{k}\left(\rho_{k}, p_{k}\right)
$$

Once the EOS is given, we can define the celerity $c_{k}$ of acoustics waves in phase $k$, and its temperature $T_{k}>0$ using the following relations:

$$
\begin{aligned}
\rho_{k} c_{k}^{2} & =\left(\partial_{p_{k}} \varepsilon_{k}\right)^{-1}\left(\frac{p_{k}}{\rho_{k}}-\rho_{k}\left(\partial_{\rho_{k}} \varepsilon_{k}\right)\right) \\
\frac{1}{T_{k}} & =\left(\partial_{p_{k}} \varepsilon_{k}\right)^{-1}\left(\partial_{p_{k}} s_{k}\right),
\end{aligned}
$$

where $s_{k}$ is the specific entropy of phase $k$ which complies with:

$$
c_{k}^{2}\left(\partial_{p_{k}} s_{k}\right)+\left(\partial_{\rho_{k}} s_{k}\right)=0 .
$$

Thanks to all those notations, governing equations of the two-fluid models read:

$$
\begin{cases}\partial_{t}\left(\alpha_{k}\right)+u_{I} \partial_{x}\left(\alpha_{k}\right) & =S_{1, k} \\ \partial_{t}\left(\alpha_{k} \rho_{k}\right)+\partial_{x}\left(\alpha_{k} \rho_{k} u_{k}\right) & =S_{2, k} \\ \partial_{t}\left(\alpha_{k} \rho_{k} u_{k}\right)+\partial_{x}\left(\alpha_{k} \rho_{k} u_{k}^{2}+\alpha_{k} p_{k}\right)-p_{I} \partial_{x}\left(\alpha_{k}\right) & =S_{3, k} \\ \partial_{t}\left(\alpha_{k} \rho_{k} e_{k}\right)+\partial_{x}\left(\alpha_{k} \rho_{k} e_{k} u_{k}+\alpha_{k} p_{k} u_{k}\right)-p_{I} u_{I} \partial_{x}\left(\alpha_{k}\right) & =S_{4, k} .\end{cases}
$$

The right-hand side terms $S_{j, k}(j=1,4)$ are so-called source terms and represent exchanges between phases. If no exterior source is considered, they comply with $S_{j, 1}+S_{j, 2}=0$. Thus the first equation in system (2.2) is redundant for both phases so those two-fluid models are seven-equation models.

The closure laws for the couple of interfacial quantities $\left(u_{I}, p_{I}\right)$ are the following ones:

$$
\begin{array}{ll}
u_{I}=a u_{1}+(1-a) u_{2}, & a=\frac{\chi m_{1}}{\chi m_{1}+(1-\chi) m_{2}}, \\
p_{I}=b p_{1}+(1-b) p_{2}, & b=\frac{(1-a) T_{2}}{a T_{1}+(1-a) T_{2}} .
\end{array}
$$

They are a generalization of those proposed by Coquel et al. $[7,16]$ which are based on two important requirements: the enforcement of a relevant entropy inequality and a linearly degenerate field associated with $\lambda=u_{I}$. Many other closure laws could be found in references $[6,20,27,38]$ and some generalisations to a larger number of components have been recently proposed $[22,33]$. The classical choice of Baer and Nunziato [3] where the 
interfacial velocity is equal to the velocity of one of the phase corresponds to $\chi=0$ or $\chi=1$. We denote respectively $\mathrm{BN} 1$ model and $\mathrm{BN} 2$ model, the case when $\left(u_{I}, p_{I}\right)=\left(u_{1}, p_{2}\right)$ and $\left(u_{I}, p_{I}\right)=\left(u_{2}, p_{1}\right)$. The case when $\chi=\frac{1}{2}$ corresponds to the model proposed in [7,16] and will be denoted as CGHS model in reference to the authors. In the sequel, we will restrict our attention to those three models $\left(\chi=0, \frac{1}{2}, 1\right)$. Another possible choice would have been to consider $\chi$ as another variable which would require another evolution law then. It leads to a class of two-fluid models that has been proposed in [23] and which is briefly presented in Appendix A.

At this stage, it only remains to define the source terms. For CGHS and BN models, we have the following relations:

$$
\begin{aligned}
S_{1, k} & =\Phi_{k}, \\
S_{2, k} & =\Gamma_{k}, \\
S_{3, k} & =D_{k}+\mathcal{U} \Gamma_{k}, \\
S_{4, k} & =Q_{k}+\mathcal{U} D_{k}+\mathcal{H} \Gamma_{k}-p_{I} \Phi_{k},
\end{aligned}
$$

where $\mathcal{U}=\frac{1}{2}\left(u_{1}+u_{2}\right)$ and $\mathcal{H}=\frac{1}{2}\left(u_{1} u_{2}\right)$. The contribution $\Phi_{k}$ verifies $\Phi_{1}+\Phi_{2}=0$, as well as the interfacial mass transfer $\Gamma_{k}$, the drag effects $D_{k}$ and the interfacial heat transfer $Q_{k}$. All those contributions are written as relaxation phenomena:

$$
\begin{aligned}
\Phi_{k} & =\frac{1}{\tau_{p} p_{\mathrm{ref}}} \alpha_{k} \alpha_{j}\left(p_{k}-p_{j}\right), \\
\Gamma_{k} & =\frac{1}{\tau_{\mu} \mu_{\mathrm{ref}}} \frac{m_{k} m_{j}}{m_{k}+m_{j}}\left(\mu_{j}-\mu_{k}\right), \quad j=3-k \\
D_{k} & =\frac{1}{\tau_{u}} \frac{m_{k} m_{j}}{m_{k}+m_{j}}\left(u_{j}-u_{k}\right), \\
Q_{k} & =\frac{1}{\tau_{T}} \frac{m_{k} C_{V k} m_{j} C_{V j}}{m_{k} C_{V k}+m_{j} C_{V j}}\left(T_{j}-T_{k}\right),
\end{aligned}
$$

where $\mu_{k}$ is the chemical potential of phase $k$ which depends on the specific Gibbs enthalpy $g_{k}$ :

$$
\mu_{k}=\frac{g_{k}}{T_{k}} \quad \text { with } \quad g_{k}=\varepsilon_{k}+\frac{p_{k}}{\rho_{k}}-T_{k} s_{k}
$$

$C_{V k}$ represents the heat capacity of phase $k, p_{\text {ref }}$ and $\mu_{\text {ref }}$ are respectively a reference pressure and a reference chemical potential. All relaxation phenomena involve relaxation time scales $\tau_{\varphi}$ which lead physical quantity $\varphi=p, \mu, u, T$ towards equilibrium. The evaluation of such time scales will be discussed later on in the paper. Readers are also refered to $[3,4]$ for slightly different forms of source terms in the field of granular reactive materials.

\subsection{Main properties of the models}

We may now recall the main properties of the three models defined earlier (BN1, BN2 and CGHS). Due to a similar construction with the same requirements, the three models share the same properties. More details on the CGHS model, which is the most difficult case, could be found in [16] with all proofs, comments and details.

\section{- Entropy inequality}

Smooth solutions of system (2.2) with closure laws of CGHS or BN models comply with an entropy inequality:

$$
\begin{aligned}
\partial_{t}\left(\sum_{k} m_{k} s_{k}\right)+\partial_{x}\left(\sum_{k} m_{k} s_{k} u_{k}\right)= & \left(\mu_{2}-\mu_{1}\right) \Gamma_{1}+\left(\frac{1-b}{T_{1}}+\frac{b}{T_{2}}\right)\left(p_{1}-p_{2}\right) \Phi_{1} \\
& +\left(\frac{1}{2 T_{1}}+\frac{1}{2 T_{2}}\right)\left(u_{2}-u_{1}\right) D_{1}+\left(\frac{1}{T_{1}}-\frac{1}{T_{2}}\right) Q_{1} \geq 0 .
\end{aligned}
$$




\section{- Hyperbolicity and structure of waves}

The convective part of system (2.2):

$$
\begin{cases}\partial_{t}\left(\alpha_{k}\right)+u_{I} \partial_{x}\left(\alpha_{k}\right) & =0 \\ \partial_{t}\left(\alpha_{k} \rho_{k}\right)+\partial_{x}\left(\alpha_{k} \rho_{k} u_{k}\right) & =0 \\ \partial_{t}\left(\alpha_{k} \rho_{k} u_{k}\right)+\partial_{x}\left(\alpha_{k} \rho_{k} u_{k}^{2}+\alpha_{k} p_{k}\right)-p_{I} \partial_{x}\left(\alpha_{k}\right) & =0 \\ \partial_{t}\left(\alpha_{k} \rho_{k} e_{k}\right)+\partial_{x}\left(\alpha_{k} \rho_{k} e_{k} u_{k}+\alpha_{k} p_{k} u_{k}\right)-p_{I} u_{I} \partial_{x}\left(\alpha_{k}\right)=0\end{cases}
$$

is hyperbolic. It admits seven real eigenvalues:

$$
\begin{aligned}
& \lambda_{1}=u_{I}, \\
& \lambda_{2}=u_{1}-c_{1}, \quad \lambda_{3}=u_{1}, \quad \lambda_{4}=u_{1}+c_{1}, \\
& \lambda_{5}=u_{2}-c_{2}, \quad \lambda_{6}=u_{2}, \quad \lambda_{7}=u_{2}+c_{2}
\end{aligned}
$$

and associated righteigenvectors span the whole space $\mathbb{R}^{7}$ if $\left|u_{k}-u_{I}\right| \neq c_{k}$. Fields associated with eigenvalues $\lambda_{1,3,6}$ are linearly degenerate (LD) whereas fields associated with eigenvalues $\lambda_{2,4,5,7}$ are genuinely non linear (GNL).

\section{- Jump conditions}

Unique jump conditions hold within each isolated field. Moreover, classical single phase jump relations hold in the GNL fields:

$$
\left\{\begin{array}{c}
{\left[\alpha_{k}\right]=0} \\
-\sigma\left[\rho_{k}\right]+\left[\rho_{k} u_{k}\right]=0 \\
-\sigma\left[\rho_{k} u_{k}\right]+\left[\rho_{k} u_{k}^{2}+p_{k}\right]=0 \\
-\sigma\left[\rho_{k} e_{k}\right]+\left[\rho_{k} e_{k} u_{k}+p_{k} u_{k}\right]=0
\end{array}\right.
$$

where $[\varphi]=\varphi_{R}-\varphi_{L}$ is the jump between the Left and Right states on each side of a shock wave traveling at speed $\sigma$.

Though BN and CGHS models have the same properties, we emphasize the fact that they are quite different from a purely convective point of view. Indeed, the structure of waves is slightly different in the case of BN models since two LD waves collapse in that case. Thus jump conditions across the 1-field highly depend on the model. We also underline that the LD structure of the 1-field is crucial in order to obtain unique jump conditions. Otherwise the computation of grid converged shock solutions may differ, depending on the inner numerical viscosity of the scheme, due to the occurence of the "true" non-conservative products (i.e. active in GNL fields). This point is not clearly addressed in the literature on two-fluid models but interesting examples can be found in [21].

\subsection{Equation Of State and relaxation time scales}

Before presenting the numerical methods, we provide remaining closure laws. So far, models have been presented using a general EOS so we need to precise the EOS used in the computations. Besides we also explain the estimations of the different relaxation time scales $\tau_{\varphi}$.

To describe steam-water transients, we used the Stiffened Gas EOS within each phase. It requires 5 thermodynamical constants $\gamma_{k}, p_{k}^{\infty}, q_{k}, C_{V k}$ and $q_{k}^{\prime}$ [30] to provide the following thermodynamical quantities:

$$
\left\{\begin{array}{l}
\varepsilon_{k}=\left(\left(\gamma_{k}-1\right) \rho_{k}\right)^{-1}\left(p_{k}+\gamma_{k} p_{k}^{\infty}\right)+q_{k} \\
T_{k}=\left(C_{V k}\left(\gamma_{k}-1\right) \rho_{k}\right)^{-1}\left(p_{k}+p_{k}^{\infty}\right) \\
s_{k}=C_{V k} \ln \left(\left(C_{V k}\left(\gamma_{k}-1\right) \rho_{k}\right)^{-\gamma_{k}}\left(p_{k}+p_{k}^{\infty}\right)\right)+q_{k}^{\prime} .
\end{array}\right.
$$


Moreover, Stiffened Gas EOS are only defined if $\gamma_{k}>1, C_{V k}>0$ and $p_{k}^{\infty}>0$. We also require $X_{k}=p_{k}+p_{k}^{\infty}>0$ in order to guarantee positivity of some thermodynamical quantities.

Evaluations of relaxation time scales are difficult to provide. Most available work in the literature deals with instantaneous relaxation $[11,31,34]$ although the underlying physical phenomena could be less fast. In our case, we only consider non-instantaneous relaxations so that we need estimations of relaxation time scales. For pressure and velocity relaxations, which should reduce the differences between CGHS and BN models, we use physical approximations of the relaxation time scales. The other two relaxation time scales are linked together and more complex to evaluate. In particular, only a few approximations of the chemical potential relaxation time scale, which drives the mass transfer, could be found in reference [13]. Moreover, those approximations only deal with homogeneous models. Therefore chemical potential and temperature relaxation time scales will be assumed constant in the present simulations.

In the case of pressure relaxation, the evaluation of the time scale is based on the Rayleigh-Plesset equation [18], which considers the evolution of a bubble in an infinite medium. It provides the following approximation:

$$
\tau_{p} p_{\text {ref }}=\left\{\begin{array}{cl}
\frac{4}{3} \eta_{2} & \text { if } \alpha_{1}<0.2 \\
\frac{4}{3} \eta_{1} & \text { if } \alpha_{1}>0.8 \\
\left(\frac{0.8-\alpha_{1}}{0.8-0.2}\right) \frac{4}{3} \eta_{2}+\left(\frac{\alpha_{1}-0.2}{0.8-0.2}\right) \frac{4}{3} \eta_{1} & \text { otherwise }
\end{array}\right.
$$

where $\eta_{k}$ is the dynamic viscosity of phase $k$.

In the case of velocity relaxation, the approach is really similar. The behavior of a bubble in an infinite medium is given by the drag equation which leads to an approximation of the relaxation time scale. In the NEPTUNE-CFD code [29], the following model is proposed for liquid-gas separated flows:

$$
D_{k}=\alpha_{k} \alpha_{j} F_{D}\left(\alpha_{1}\right)\left(u_{j}-u_{k}\right), \quad j=3-k
$$

where the function $F_{D}$ of $\alpha_{1}$ is defined as follows:

$$
F_{D}\left(\alpha_{1}\right)=\left\{\begin{array}{cl}
F_{D, 1}\left(1-\alpha_{1}\right) & \text { if } \alpha_{1}<0.2 \\
F_{D, 2}\left(\alpha_{1}\right) & \text { if } \alpha_{1}>0.8 \\
\left(\frac{0.8-\alpha_{1}}{0.8-0.2}\right) F_{D, 1}(1-0.2)+\left(\frac{\alpha_{1}-0.2}{0.8-0.2}\right) F_{D, 2}(0.8) & \text { otherwise. }
\end{array}\right.
$$

The function $F_{D}$ is equal to the function $F_{D, p}\left(\alpha_{q}\right)$ in the case of isolated spherical inclusions of phase $p$ in the continuous phase $q$. The following classic correlations are used [29,36]:

$$
F_{D, p}\left(\alpha_{q}\right)=\frac{3}{4} \frac{\rho_{q}}{\alpha_{q}} \frac{C_{D, p}}{d_{p}}\left|u_{p}-u_{q}\right|, \quad q=3-p
$$

with:

$$
C_{D, p}=\frac{24}{R e_{p}}\left(1+0.15\left(R e_{p}\right)^{0.687}\right) \quad \text { and } \quad R e_{p}=\frac{\rho_{q} d_{p}}{\eta_{q}}\left|u_{p}-u_{q}\right|,
$$

where $d_{p}$ is a characteristical diameter of the inclusion that needs to be defined in [29]. In our case, we choose to define it using the critical Weber number $W e=10$ (see [36]):

$$
W e=\frac{\rho_{q} d_{p}}{\sigma}\left|u_{p}-u_{q}\right|^{2},
$$

where $\sigma$ is the surface tension of the liquid phase. Thus, we obtain the following approximation for the velocity relaxation time scale using (2.6) and (2.13):

$$
\tau_{u}=\frac{\rho_{1} \rho_{2}}{m_{1}+m_{2}} \frac{1}{F_{D}\left(\alpha_{1}\right)} .
$$




\section{Numerical METHOdS}

An entropy-consistent fractional step method is used to compute approximations of the whole system. This algorithm was first detailed in [24] for BN models without mass transfer. Two steps are used to compute approximate solutions of system (2.2). The first step is an evolution step where we compute approximate solutions of the homogeneous system:

$$
\begin{cases}\partial_{t}\left(\alpha_{k}\right)+u_{I} \partial_{x}\left(\alpha_{k}\right) & =0 \\ \partial_{t}\left(\alpha_{k} \rho_{k}\right)+\partial_{x}\left(\alpha_{k} \rho_{k} u_{k}\right) & =0 \\ \partial_{t}\left(\alpha_{k} \rho_{k} u_{k}\right)+\partial_{x}\left(\alpha_{k} \rho_{k} u_{k}^{2}+\alpha_{k} p_{k}\right)-p_{I} \partial_{x}\left(\alpha_{k}\right) & =0 \\ \partial_{t}\left(\alpha_{k} \rho_{k} e_{k}\right)+\partial_{x}\left(\alpha_{k} \rho_{k} e_{k} u_{k}+\alpha_{k} p_{k} u_{k}\right)-p_{I} u_{I} \partial_{x}\left(\alpha_{k}\right) & =0\end{cases}
$$

through the time interval $\left[t^{n}, t^{n}+\Delta t\right]$ with given initial values $\varphi^{n}$ of the seven variables. Thus, we obtain approximations $\widetilde{\varphi}$ which are used as initial values in the second step of the method. This second step is called relaxation step and takes into account the source terms. We compute approximate solutions of the following system:

$$
\begin{cases}\partial_{t}\left(\alpha_{k}\right) & =S_{1, k} \\ \partial_{t}\left(\alpha_{k} \rho_{k}\right) & =S_{2, k} \\ \partial_{t}\left(\alpha_{k} \rho_{k} u_{k}\right) & =S_{3, k} \\ \partial_{t}\left(\alpha_{k} \rho_{k} e_{k}\right) & =S_{4, k}\end{cases}
$$

with $S_{j, k}$ defined by equations (2.5) and (2.6). Finally, we obtain the approximations $\varphi^{n+1}$. As in [24], the relaxation time step is divided into different substeps to treat the different relaxation phenomena. In the sequel, we detail all steps and substeps of the method and we respect the order of the algorithm to present them.

\subsection{Evolution step}

The first step of the fractional step method is an evolution one. It consists in using finite volume methods to solve the convective system (3.1). In the case of the BN models, several finite volume schemes have been proposed up to now. Many of them are approximate or exact Riemann solvers. Schwendeman et al. [39] propose a Godunov scheme that approximates the LD 1-wave as a layer of vaninshingly small thickness across which the solution is assumed to be smooth. This hypothesis has been also used by Tokareva and Toro [41] to build an HLLC-type Riemann solver. Deledicque and Papalexandris [12] as well as Andrianov and Warnecke [2] propose exact Riemann solvers based on different approaches. Relaxation solvers have also been used in [8] to handle vanishing phases.

In the case of the CGHS model, very few solvers could be found in the literature. Relaxation techniques have been applied in [1] and extensions of both Rusanov and VFRoe schemes to non-conservative systems have been proposed in [16]. Among those three schemes, we choose to use a Rusanov scheme for all models for simplicity.

Moreover, grid convergence studies of the Rusanov scheme for BN model can be found in [9] where first-order and second-order schemes have been verified. The expected rate of convergence which is $1 / 2$ in the case of the first-order scheme (and 2/3 in the case of the second-order scheme) is retrieved. Those rates of convergence are due to the occurence of contact discontinuities in the exact solutions. In the case of smooth solutions, the first-order (or second-order) should be obtained. We underline the fact that only a few grid convergence studies in order to verify convective schemes could be found in reference $[8,21,24]$. Thus, we propose to carry out a grid convergence study on a Riemann problem to verify the Rusanov scheme for the CGHS model. We set particular thermodynamical parameters for both phases (see Tab. 1) in order to obtain an analytical solution. Once the Left state is set, the Right state is calculated so that the Riemann invariants of the LD 1-wave are preserved, leading to the initial states recalled in Table 2. Therefore the analytical solution of this first Riemann 
TABLE 1. EOS parameters for the first Riemann problem.

\begin{tabular}{cccccc}
\hline & $\gamma_{k}$ & $\begin{array}{c}p_{k}^{\infty} \\
(\mathrm{Pa})\end{array}$ & $\begin{array}{c}q_{k} \\
\left(\mathrm{~J} \mathrm{~kg}^{-1}\right)\end{array}$ & $\begin{array}{c}C_{V k} \\
\left(\mathrm{~J} \mathrm{~kg}^{-1} \mathrm{~K}^{-1}\right)\end{array}$ & $\begin{array}{c}q_{k}^{\prime} \\
\left(\mathrm{J} \mathrm{kg}^{-1} \mathrm{~K}^{-1}\right)\end{array}$ \\
\hline Phase 1 & 2. & 10000.00 & 2000000.00 & 1500.00 & 2000.00 \\
Phase 2 & 2. & 200000.00 & 1000.00 & 1500.00 & 25000.00 \\
\hline
\end{tabular}

TABLE 2. Initial conditions for the first Riemann problem.

\begin{tabular}{lccccc}
\hline & & $\alpha_{k}$ & $\begin{array}{c}\rho_{k} \\
\left(\mathrm{~kg} \mathrm{~m}^{-3}\right)\end{array}$ & $\begin{array}{c}p_{k} \\
(\mathrm{~Pa})\end{array}$ & $\begin{array}{c}u_{k} \\
\left(\mathrm{~m} \mathrm{~s}^{-1}\right)\end{array}$ \\
\hline \multirow{2}{*}{ Left state } & Phase 1 & 0.8 & 2.00 & 100000.00 & 100.00 \\
& Phase 2 & 0.2 & 1000.00 & 300000.00 & 100.00 \\
\hline \multirow{2}{*}{ Right state } & Phase 1 & 0.3 & 1.47780679 & 71279.3734 & 100.00 \\
& Phase 2 & 0.7 & 738.903394 & 169451.697 & 100.00 \\
\hline
\end{tabular}

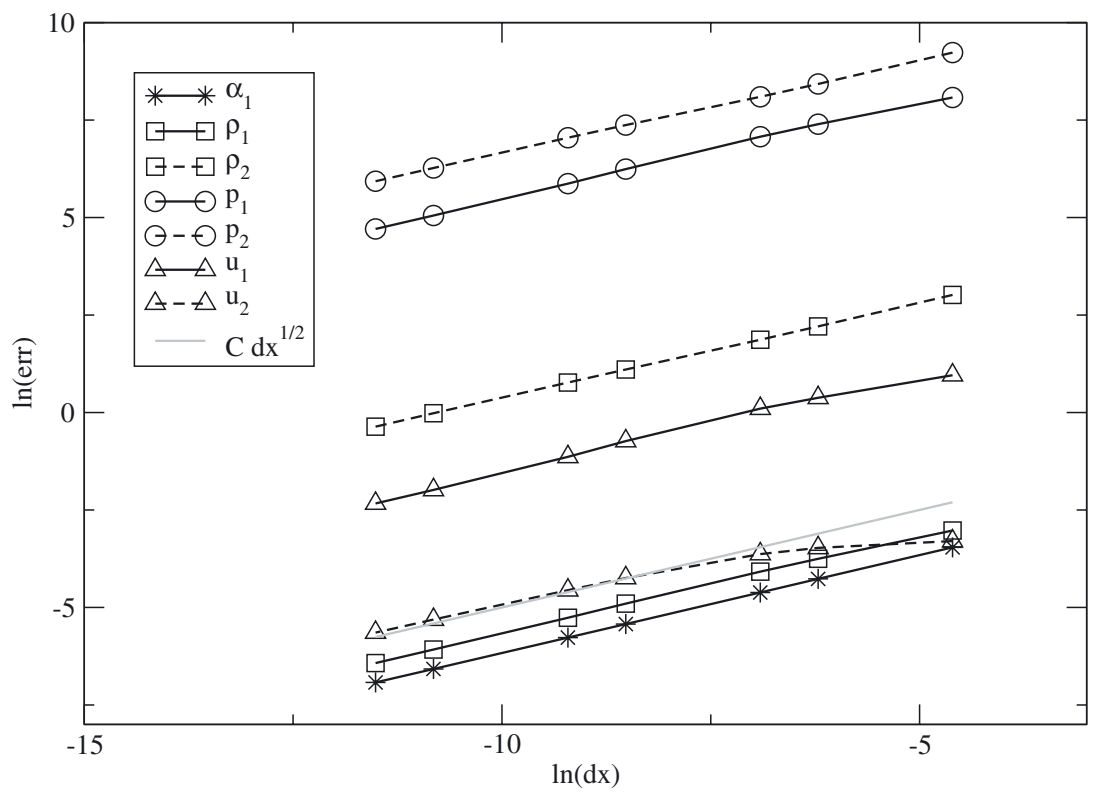

FiguRE 1. Convergence study of the Rusanov scheme for the CGHS model on the first Riemann problem: $L 1$ norm of the error vs. $\Delta x$ at $t_{f}=1.5 \times 10^{-3} \mathrm{~s}$ with a 0.5 CFL condition.

problem only exhibits the LD 1-wave. Figure 1 shows the results of the grid convergence study and we retrieve the expected rate of convergence $1 / 2$ as explained before.

Once again, the LD-structure of the 1-field should be highlighted. Indeed, due to this property, the nonconservative products are not active in GNL fields and so the three models exhibit unique jump conditions. It is of major importance to obtain unique analytical solutions of Riemann problems so that convective schemes could be verified.

As we emphasize it earlier, the three models are quite different when looking at the convective part of the models which is exactly the part we solve in the evolution step. An interesting test case proposed in [1] (Test 3) gives an illustration of those differences. This test case is a Riemann problem with two gases. Thermodynamical parameters of those gases are recalled in Table 3 and both initial states of the test case are presented in Table 4 . 
TABLE 3. EOS parameters for the second Riemann problem.

\begin{tabular}{cccccc}
\hline & $\gamma_{k}$ & $\begin{array}{c}p_{k}^{\infty} \\
(\mathrm{Pa})\end{array}$ & $\begin{array}{c}q_{k} \\
\left(\mathrm{~J} \mathrm{~kg}^{-1}\right)\end{array}$ & $\begin{array}{c}C_{V k} \\
\left(\mathrm{~J} \mathrm{~kg}^{-1} \mathrm{~K}^{-1}\right)\end{array}$ & $\begin{array}{c}q_{k}^{\prime} \\
\left(\mathrm{J} \mathrm{kg}^{-1} \mathrm{~K}^{-1}\right)\end{array}$ \\
\hline Phase 1 & 1.4 & 0.00 & 0.00 & 1000.00 & 0.00 \\
Phase 2 & 1.4 & 0.00 & 0.00 & 1000.00 & 0.00 \\
\hline
\end{tabular}

TABLE 4. Initial conditions for the second Riemann problem.

\begin{tabular}{llcccc}
\hline & & $\alpha_{k}$ & $\begin{array}{c}\rho_{k} \\
\left(\mathrm{~kg} \mathrm{~m}^{-3}\right)\end{array}$ & $\begin{array}{c}p_{k} \\
(\mathrm{~Pa})\end{array}$ & $\begin{array}{c}u_{k} \\
\left(\mathrm{~m} \mathrm{~s}^{-1}\right)\end{array}$ \\
\hline \multirow{2}{*}{ Left state } & Phase 1 & 0.8 & 1.00 & 1.00 & 0.00 \\
& Phase 2 & 0.2 & 0.20 & 0.30 & 0.00 \\
\hline \multirow{2}{*}{ Right state } & Phase 1 & 0.3 & 1.00 & 1.00 & 0.00 \\
& Phase 2 & 0.7 & 1.00 & 1.00 & 0.00 \\
\hline
\end{tabular}

The numerical results given by first-order Rusanov schemes with a CFL condition of 0.5 on a 1D mesh made of 1000 cells are presented in Figure 2. As expected, the differences between the models are striking. The intermediate states are totally different in some cases. We can also notice that wave speeds are different as well. Moreover, the variable $\rho_{2}$ is a good example to underline the fact that the models don't have the same number of waves. In the case of the CGHS model, $\rho_{2}$ is an invariant of 3 waves (the three waves on Phase 1) among the 7 waves of the model which gives us the 4 waves (and the 3 intermediate states) that can be observed on $\rho_{2}$. For the BN2 model, $\rho_{2}$ is also an invariant of 3 waves but the model has only 6 waves so this variable exhibits 3 waves. To finish, $\rho_{2}$ is an invariant of only 2 waves of the BN1 model (the 2 GNL waves on Phase 1) so its leads to the 4 waves we can see on $\rho_{2}$ in Figure 2.

\subsection{Relaxation step}

The relaxation step is associated to the treatment of the source terms. During that step, we solve system (3.2) with 4 substeps, one for each relaxation phenomenum. They are treated in the following order: pressure relaxation, velocity relaxation, temperature relaxation and chemical potential relaxation. For each substep, we use the approach proposed in [24] for BN models. Thus, implicit first-order Euler schemes are used with the time step given by the CFL condition linked to the evolution step. We underline the fact that applying this approach in the case of the CGHS model requires a specific treatment in the case of the pressure relaxation. Moreover, we extend all schemes to the case when both phases are governed by Stiffened Gas EOS.

In the sequel, we use the notation $\varphi^{0}$ to denote the state of a variable $\varphi$ at the end of the previous substep and $\varphi^{*}$ the state of the same variable at the end of the current substep. $\Delta t$ denotes the time step given by the CFL condition associated to the evolution step.

\subsubsection{Pressure relaxation}

In this substep, we compute approximate solutions of the system:

$$
\begin{cases}\partial_{t}\left(\alpha_{k}\right) & =\Phi_{k} \\ \partial_{t}\left(\alpha_{k} \rho_{k}\right) & =0 \\ \partial_{t}\left(\alpha_{k} \rho_{k} u_{k}\right) & =0 \\ \partial_{t}\left(\alpha_{k} \rho_{k} e_{k}\right) & =-p_{I} \Phi_{k}\end{cases}
$$



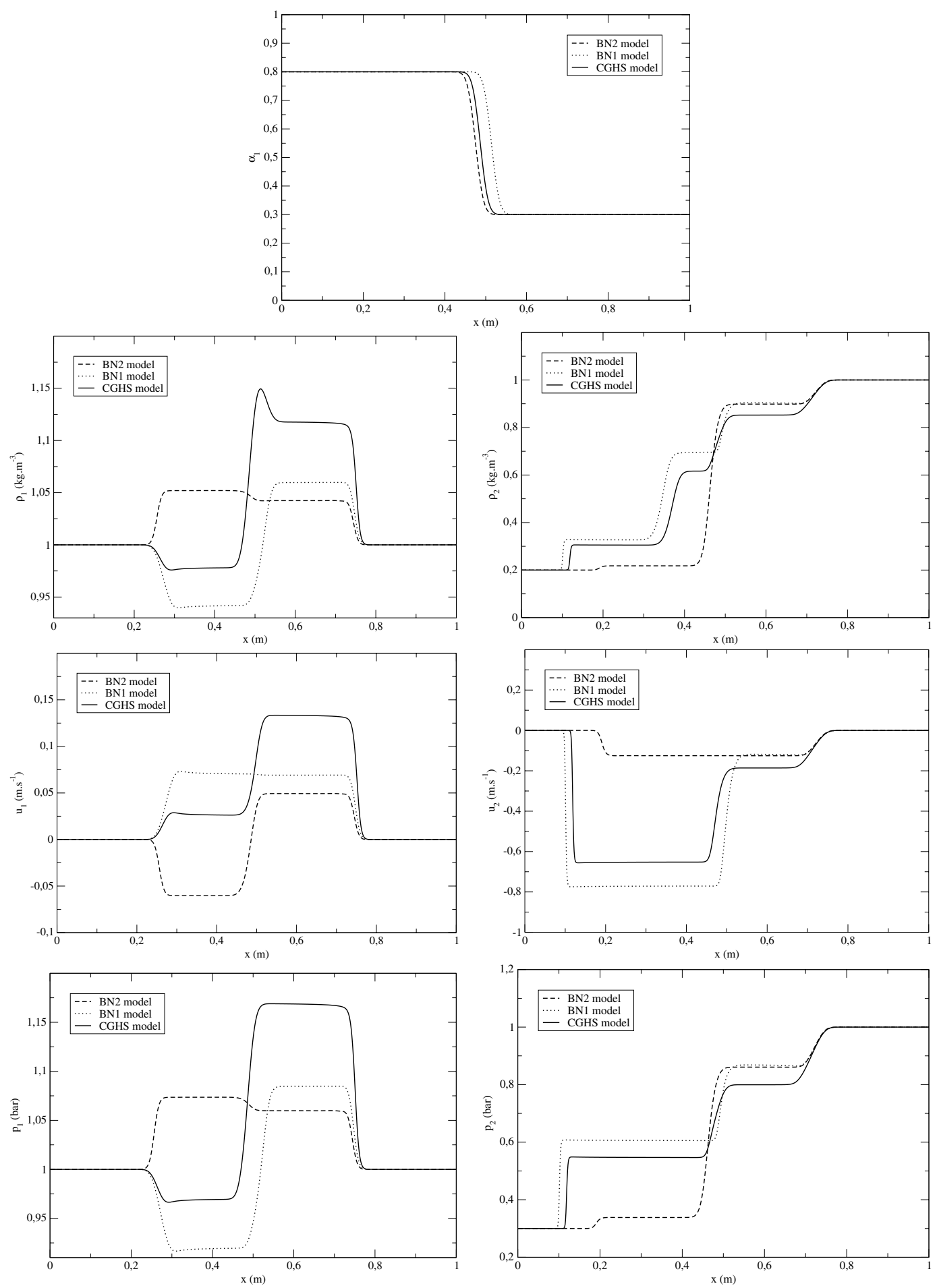

Figure 2. Comparison between numerical solutions of the three models in the second Riemann problem at time $t=0.2 \mathrm{~s}$ on a 1000-cell mesh. From the top to bottom right: $\alpha_{1}, \rho_{1}, \rho_{2}, u_{1}, u_{2}, p_{1}, p_{2}$ vs. $x$. 
Because of the occurence of the interfacial pressure $p_{I}$, the choice of the model has a major impact in this substep. We propose the following implicit scheme to solve system (3.3):

$$
\left\{\begin{array}{rl}
\frac{\alpha_{k}^{*}-\alpha_{k}^{0}}{\Delta t} & =\frac{1}{\left(\tau_{p} p_{\text {ref }}\right)} \alpha_{k}^{*} \alpha_{j}^{*}\left(p_{k}^{*}-p_{j}^{*}\right), \\
m_{k}^{*} & =m_{k}^{0}, \\
u_{k}^{*} & =u_{k}^{0},
\end{array} \quad j=3-k\right.
$$

where the interfacial pressure $p_{I}$ defined by $(2.4)$ is treated in a semi-implicit way. Therefore, the following non-linear system in $\alpha_{1}^{*}, p_{1}^{*}$ and $p_{2}^{*}$ has to be solved in the case of Stiffened Gas:

$$
\left\{\begin{aligned}
\frac{\left(\tau_{p} p_{\mathrm{ref}}\right)^{0}}{\Delta t} \frac{\alpha_{1}^{*}-\alpha_{1}^{0}}{\alpha_{1}^{*}\left(1-\alpha_{1}^{*}\right)}-\left(p_{1}^{*}-p_{2}^{*}\right) & =0 \\
\gamma_{1}\left(\alpha_{1}^{*}-\alpha_{1}^{m}\right) X_{1}^{*}-\alpha_{1}^{0} X_{1}^{0} & =\left(1-b^{0}\right)\left(\gamma_{1}-1\right)\left(\alpha_{1}^{*}-\alpha_{1}^{0}\right)\left(p_{1}^{*}-p_{2}^{*}\right), \\
-\gamma_{2}\left(\alpha_{1}^{*}-\alpha_{1}^{M}\right) X_{2}^{*}-\alpha_{2}^{0} X_{2}^{0} & =b^{0}\left(\gamma_{2}-1\right)\left(\alpha_{1}^{*}-\alpha_{1}^{0}\right)\left(p_{1}^{*}-p_{2}^{*}\right)
\end{aligned}\right.
$$

where we use the following notations:

$$
\begin{aligned}
X_{k} & =p_{k}+p_{k}^{\infty}, \\
\alpha_{1}^{m} & =\frac{\left(\gamma_{1}-1\right) \alpha_{1}^{0}}{\gamma_{1}} \quad \text { and } \quad \alpha_{1}^{M}=1-\frac{\left(\gamma_{2}-1\right) \alpha_{2}^{0}}{\gamma_{2}} .
\end{aligned}
$$

Equations (3.5b) and (3.5c) form a linear system in $p_{1}^{*}$ and $p_{2}^{*}$. Solving this system makes it possible to write those pressures as functions of $\alpha_{1}^{*}$. Thus equation (3.5a) could be written as a non-linear equation on $\alpha_{1}^{*}$ :

$$
G\left(\alpha_{1}^{*}\right)=0,
$$

where the function $G$ is defined as follows:

$$
G\left(\alpha_{1}^{*}\right)=\frac{\left(\tau_{p} p_{\mathrm{ref}}\right)^{0}}{\Delta t} \frac{\alpha_{1}^{*}-\alpha_{1}^{0}}{\alpha_{1}^{*}\left(1-\alpha_{1}^{*}\right)}-\left(p_{1}^{*}\left(\alpha_{1}^{*}\right)-p_{2}^{*}\left(\alpha_{1}^{*}\right)\right) .
$$

Due to the non-linearities of the function $G$, we use an iterative procedure to solve this equation. Particular attention is required to ensure that thermodynamical quantities remain in the admissible range. We can notice that the linear system in $p_{1}^{*}$ and $p_{2}^{*}$ made of equations $(3.5 \mathrm{~b})$ and $(3.5 \mathrm{c})$ is easier to solve in the case of $\mathrm{BN}$ models than in the case of the CGHS model. In the sequel, we will examine existence and uniqueness of solution of equation (3.7) in the case of the CGHS model (i.e. $\left.b^{0} \in\right] 0,1[$ ). Same results hold for the BN models.

Existence of a solution for equation (3.7) is guaranteed by the following:

Proposition 3.1. Assume that $p_{1}^{*}\left(\alpha_{1}^{*}\right)$ and $p_{2}^{*}\left(\alpha_{1}^{*}\right)$ are continuous functions. The equation $G\left(\alpha_{1}^{*}\right)=0$ has at least a solution in the interval $] \alpha_{1}^{m}, \alpha_{1}^{M}\left[\right.$ and this solution leads to admissible pressures $\left(X_{1}^{*}>0\right.$ and $\left.X_{2}^{*}>0\right)$.

Proof. Let us first remark the following results:

$$
\alpha_{1}^{m}-\alpha_{1}^{0}=-\frac{\alpha_{1}^{0}}{\gamma_{1}}<0 \quad \text { and } \quad \alpha_{1}^{M}-\alpha_{1}^{0}=\frac{\alpha_{2}^{0}}{\gamma_{2}}>0
$$


which also gives $\alpha_{1}^{m}<\alpha_{1}^{M}$. Using (3.9), the equation (3.5b) gives $\left(p_{1}^{*}\left(\alpha_{1}^{m}\right)-p_{2}^{*}\left(\alpha_{1}^{m}\right)\right)>0$. We deduce that $G\left(\alpha_{1}^{m}\right)<0$ by using equation (3.9) again. The same strategy applied on equation (3.5c) gives $G\left(\alpha_{1}^{M}\right)>0$ and we obtain that it exists $\left.\alpha_{1}^{*} \in\right] \alpha_{1}^{m}, \alpha_{1}^{M}\left[\right.$ such that $G\left(\alpha_{1}^{*}\right)=0$.

The admissibility of pressures is now easy to prove. Equation $(3.5 \mathrm{a})$ shows that $\left(p_{1}^{*}-p_{2}^{*}\right)$ and $\left(\alpha_{1}^{*}-\alpha_{1}^{0}\right)$ have the same sign. Hence, the right hand sides of equations (3.5b) and (3.5c) are positive. Therefore, if $\left.\alpha_{1}^{*} \in\right] \alpha_{1}^{m}, \alpha_{1}^{M}\left[\right.$, then $X_{1}^{*}>0$ and $X_{2}^{*}>0$.

Proposition 3.2. If we also assume that functions $p_{k}^{*}\left(\alpha_{k}^{*}\right)$ are strictly decreasing, then equation $G\left(\alpha_{1}^{*}\right)=0$ has a unique solution in $] \alpha_{1}^{m}, \alpha_{1}^{M}[$.

Proof. Thanks to the hypothesis on $p_{k}^{*}\left(\alpha_{k}^{*}\right)$, the function $\left(p_{1}^{*}\left(\alpha_{1}^{*}\right)-p_{2}^{*}\left(\alpha_{1}^{*}\right)\right)$ is decreasing. Let's now remark that function $\frac{\alpha_{1}^{*}-\alpha_{1}^{0}}{\alpha_{1}^{*}\left(1-\alpha_{1}^{*}\right)}$ is strictly increasing on $] 0,1\left[\right.$ and we can deduce that the function $G\left(\alpha_{1}^{*}\right)$ is strictly increasing on $] \alpha_{1}^{m}, \alpha_{1}^{M}[$. Therefore, it admits a unique zero on this interval.

In practice, the linear system in $\left(p_{1}^{*}, p_{2}^{*}\right)$ composed by equations $(3.5 \mathrm{~b})$ and $(3.5 \mathrm{c})$ can be easily solved in the case of two perfect gas $\left(p_{k}^{\infty}=0\right)$. Then, we can directly check that functions $p_{k}^{*}\left(\alpha_{k}^{*}\right)$ are strictly decreasing in that particular case. The present scheme has been verified, details can be found in Appendix B.

\subsubsection{Velocity relaxation}

The velocity relaxation substep computes approximations of solutions of the following system:

$$
\left\{\begin{array}{l}
\partial_{t}\left(\alpha_{k}\right)=0 \\
\partial_{t}\left(m_{k}\right)=0, \\
\partial_{t}\left(m_{k} u_{k}\right)=D_{k}, \\
\partial_{t}\left(m_{k} e_{k}\right)=\mathcal{U} D_{k} .
\end{array}\right.
$$

Solving this system is easier than solving the one of the previous substep. Indeed, we can show that:

$$
\partial_{t}\left(u_{1}-u_{2}\right)=-\frac{1}{\tau_{u}}\left(u_{1}-u_{2}\right) .
$$

Hence, if $\tau_{u}$ is assumed constant, analytical solution of system (3.10) could be provided. Therefore we propose the following numerical scheme, where $\tau_{u}$ is treated in an explicit way during the time interval $\left[t^{n}, t^{n}+\Delta t\right]$ :

$$
\left\{\begin{array}{l}
\alpha_{k}^{*}=\alpha_{k}^{0}, \\
m_{k}^{*}=m_{k}^{0}, \\
u_{k}^{*}=u_{k}^{0}-\frac{m_{j}^{0}}{m_{k}^{0}+m_{j}^{0}}\left(1-\mathrm{e}^{-\Delta t / \tau_{u}^{0}}\right)\left(u_{k}^{0}-u_{j}^{0}\right), \quad j=3-k \\
\varepsilon_{k}^{*}=\varepsilon_{k}^{0}+\frac{1}{4} \frac{m_{j}^{0}}{m_{k}^{0}+m_{j}^{0}}\left(1-\mathrm{e}^{-2 \Delta t / \tau_{u}^{0}}\right)\left(u_{k}^{0}-u_{j}^{0}\right)^{2} .
\end{array}\right.
$$

Note that the internal energy remains positive during this step. Moreover the relaxation effect on the velocities is ensured without any constraint on the time step. The verification of such a scheme can be found in [24]. 


\subsubsection{Temperature relaxation}

In this substep, we compute approximate solutions of the following system:

$$
\left\{\begin{array}{l}
\partial_{t}\left(\alpha_{k}\right)=0, \\
\partial_{t}\left(\alpha_{k} \rho_{k}\right)=0, \\
\partial_{t}\left(\alpha_{k} \rho_{k} u_{k}\right)=0, \\
\partial_{t}\left(\alpha_{k} \rho_{k} e_{k}\right)=Q_{k} .
\end{array}\right.
$$

As in the previous substep, the relaxation phenomenum is given by a simple equation:

$$
\partial_{t}\left(T_{1}-T_{2}\right)=-\frac{1}{\tau_{T}}\left(T_{1}-T_{2}\right)
$$

Thus, we use the following numerical scheme:

$$
\left\{\begin{array}{l}
\alpha_{k}^{*}=\alpha_{k}^{0}, \\
m_{k}^{*}=m_{k}^{0}, \\
u_{k}^{*}=u_{k}^{0}, \\
T_{k}^{*}=T_{k}^{0}-\frac{m_{j}^{0} C_{V j}}{m_{k}^{0} C_{V k}+m_{j}^{0} C_{V j}}\left(1-\mathrm{e}^{-\Delta t / \tau_{T}^{0}}\right)\left(T_{k}^{0}-T_{j}^{0}\right)
\end{array}\right.
$$

We underline the fact that the temperatures remain positive and their relaxation is ensured without any constraint on the time step. This numerical scheme corresponds to the one proposed in [24] in the case of Stiffened Gas EOS.

\subsubsection{Chemical potential relaxation}

The last substep corresponds to the numerical treatment of the mass transfer. We compute approximate solutions of the system:

$$
\left\{\begin{array}{l}
\partial_{t}\left(\alpha_{k}\right)=0 \\
\partial_{t}\left(\alpha_{k} \rho_{k}\right)=\Gamma_{k}, \\
\partial_{t}\left(\alpha_{k} \rho_{k} u_{k}\right)=\mathcal{U} \Gamma_{k}, \\
\partial_{t}\left(\alpha_{k} \rho_{k} e_{k}\right)=\mathcal{H} \Gamma_{k} .
\end{array}\right.
$$

Thanks to simple manipulations, it could be written in the following way:

$$
\left\{\begin{array}{l}
\partial_{t}\left(\alpha_{k}\right)=0 \\
\partial_{t}\left(m_{k}\right)=\Gamma_{k}, \\
\partial_{t}\left(m_{k} u_{k}\right)=\mathcal{U} \Gamma_{k}, \\
\partial_{t}\left(m_{k} \varepsilon_{k}\right)=0
\end{array}\right.
$$


An implicit scheme is used to approximate solutions of that system, which is:

$$
\left\{\begin{array}{l}
\alpha_{k}^{*}=\alpha_{k}^{0}, \\
\frac{m_{k}^{*}-m_{k}^{0}}{\Delta t}=\frac{1}{\left(\tau_{\mu} \mu_{\mathrm{ref}}\right)^{0}} \frac{m_{k}^{*} m_{j}^{*}}{m_{k}^{*}+m_{j}^{*}}\left(\mu_{j}^{*}-\mu_{k}^{*}\right), \\
\frac{m_{k}^{*} u_{k}^{*}-m_{k}^{0} u_{k}^{0}}{\Delta t}=\frac{u_{k}^{*}+u_{j}^{*}}{2} \frac{m_{k}^{*}-m_{k}^{0}}{\Delta t}, \\
m_{k}^{*} \varepsilon_{k}^{*}=m_{k}^{0} \varepsilon_{k}^{0} .
\end{array}\right.
$$

Equation (3.16b) is written as follows:

$$
H\left(m_{1}^{*}\right)=0
$$

where we use the following notations:

$$
\begin{aligned}
H\left(m_{1}^{*}\right) & =M_{T} \frac{\left(\tau_{\mu} \mu_{\mathrm{ref}}\right)^{0}}{\Delta t} \frac{m_{1}^{*}-m_{1}^{0}}{m_{1}^{*}\left(M_{T}-m_{1}^{*}\right)}+\left(\mu_{1}^{*}\left(m_{1}^{*}\right)-\mu_{2}^{*}\left(M_{T}-m_{1}^{*}\right)\right), \\
M_{T} & =m_{1}^{0}+m_{2}^{0}=m_{1}^{*}+m_{2}^{*} .
\end{aligned}
$$

Chemical potentials $\mu_{k}^{*}$ may be seen as functions of $m_{k}^{*}$ thanks to equations (3.16a) and (3.16d). Indeed, we have:

$$
\mu_{k}^{*}=\mu_{k}^{*}\left(\rho_{k}^{*}, \varepsilon_{k}^{*}\right)=\mu_{k}^{*}\left(\frac{m_{k}^{*}}{\alpha_{k}^{0}}, \frac{m_{k}^{0} \varepsilon_{k}^{0}}{m_{k}^{*}}\right) .
$$

Once equation (3.17) has been solved, velocities $u_{k}^{*}$ are calculated by solving the linear system made of equation (3.16c) for both phases. Its determinant is strictly positive: $\Delta=\frac{1}{2}\left(m_{1}^{0} m_{2}^{*}+m_{2}^{0} m_{1}^{*}\right)$. Thus, the main difficulty of the scheme lies in solving non-linear equation (3.17). In the numerical scheme, this difficulty is overcomed by using an iterative solver. As it happens for the pressure relaxation, specific attention is required to ensure that thermodynamical quantities remain in the admissible range. This is guaranteed by the following property:

Proposition 3.3. The equation $H\left(m_{1}^{*}\right)=0$ has a unique solution with positive partial masses and temperatures.

Proof. First, we focus on the constraint on the signs of the temperatures. Let us first express temperatures as functions of partial masses thanks to the EOS (2.11):

$$
T_{k}^{*}\left(m_{k}^{*}\right)=\frac{1}{C_{V k}}\left(\frac{m_{k}^{0} \varepsilon_{k}^{0}-\alpha_{k}^{0} p_{k}^{\infty}}{m_{k}^{*}}-q_{k}\right) .
$$

If $q_{k} \neq 0$, we introduce $\varphi_{k}$ as follows:

$$
\varphi_{k}=\frac{m_{k}^{0} \varepsilon_{k}^{0}-\alpha_{k}^{0} p_{k}^{\infty}}{q_{k}}
$$

It results in the following expressions of temperatures:

$$
T_{k}^{*}\left(m_{k}^{*}\right)=\frac{q_{k}}{C_{V k} m_{k}^{*}}\left(\varphi_{k}-m_{k}^{*}\right) .
$$

So we can define the intervals $I_{k}$ that ensure that temperatures $T_{k}^{*}$ remain positive:

$$
I_{1}=\left\{\begin{array}{c}
] 0, \varphi_{1}\left[\text { if } q_{1}>0\right. \\
] 0,+\infty\left[\text { if } q_{1}=0\right. \\
] \varphi_{1}^{+},+\infty\left[\text { if } q_{1}<0\right.
\end{array} \quad \text { and } \quad I_{2}=\left\{\begin{aligned}
]\left(M_{T}-\varphi_{2}\right)^{+},+\infty[ & \text { if } q_{2}>0 \\
] 0,+\infty[ & \text { if } q_{2}=0 \\
] 0,\left(M_{T}-\varphi_{2}\right)[ & \text { if } q_{2}<0
\end{aligned}\right.\right.
$$


where we use the classical notation $\psi^{+}=\max (0, \psi)$. Then we define the interval $] m_{1}^{\min }, m_{1}^{\max }$ [ that ensure that all temperatures and partial masses remain positive:

$$
] m_{1}^{\min }, m_{1}^{\max }[=] 0, M_{T}\left[\cap I_{1} \cap I_{2} .\right.
$$

In each case, depending on the signs of $q_{k}$, we can prove that $] m_{1}^{\min }, m_{1}^{\max }[\neq \emptyset$. We skip the details for simplicity but we can also prove the following limits:

$$
\lim _{m_{1}^{*} \rightarrow m_{1}^{\min }}\left(\mu_{1}^{*}\left(m_{1}^{*}\right)-\mu_{2}^{*}\left(M_{T}-m_{1}^{*}\right)\right)=-\infty \quad \text { and } \quad \lim _{m_{1}^{*} \rightarrow m_{1}^{\max }}\left(\mu_{1}^{*}\left(m_{1}^{*}\right)-\mu_{2}^{*}\left(M_{T}-m_{1}^{*}\right)\right)=+\infty
$$

where:

$$
\begin{aligned}
\mu_{k}^{*}\left(m_{k}^{*}\right)= & \gamma_{k} C_{V k}+\frac{q_{k}}{T_{k}^{*}\left(m_{k}^{*}\right)}-C_{V k} \ln \left(T_{k}^{*}\left(m_{k}^{*}\right)\right)+\left(\gamma_{k}-1\right) C_{V k} \ln \left(m_{k}^{*}\right) \\
& +\left(\gamma_{k}-1\right) C_{V k} \ln \left(\left(\gamma_{k}-1\right) C_{V k} \frac{1}{\alpha_{k}^{0}}\right)-q_{k}^{\prime} .
\end{aligned}
$$

Then, it is easy to deduce the limits of the function $H\left(m_{1}^{*}\right)$ :

$$
\lim _{m_{1}^{*} \rightarrow m_{1}^{\min }} H\left(m_{1}^{*}\right)=-\infty \text { and } \lim _{m_{1}^{*} \rightarrow m_{1}^{\max }} H\left(m_{1}^{*}\right)=+\infty .
$$

Hence, the continuous function $H\left(m_{1}^{*}\right)$ has at least one zero in $] m_{1}^{\min }, m_{1}^{\max }[$.

Then, we focus on the monotonicity of $H$ in order to prove that such a zero is unique. We have the following derivative:

$$
\mu_{k}^{* \prime}\left(m_{k}^{*}\right)=\frac{\left(\gamma_{k}-1\right) C_{V k}}{m_{k}^{*}}+\frac{1}{C_{V k}\left(T_{k}^{*}\left(m_{k}^{*}\right)\right)^{2}} \frac{\left(m_{k}^{0} \varepsilon_{k}^{0}-\alpha_{k}^{0} p_{k}^{\infty}\right)^{2}}{m_{k}^{* 3}} .
$$

So the function $\left(\mu_{1}^{*}\left(m_{1}^{*}\right)-\mu_{2}^{*}\left(M_{T}-m_{1}^{*}\right)\right)$ is strictly increasing on $] m_{1}^{\min }, m_{1}^{\max }$ [. Moreover, the function $\frac{m_{1}^{*}-m_{1}^{0}}{m_{1}^{*}\left(M_{T}-m_{1}^{*}\right)}$ is also strictly increasing on $] 0, M_{T}\left[\right.$. Thus, $H\left(m_{1}^{*}\right)$ is strictly increasing on $] m_{1}^{\min }, m_{1}^{\max }[$ and the equation $H\left(m_{1}^{*}\right)=0$ has a unique solution in that interval.

Appendix C provides a verification of the scheme. Note that the present scheme is an extension of the one proposed in [10] for Stiffened Gas EOS.

\section{Comparison OF TWO-FLUid MODELS ON STEAM-WATER TRANSIENTS}

This section is devoted to the comparison of the three different models described earlier. We focus in this paper on steam-water applications involving mass transfer. Two experimental configurations have been selected so that we could compare models with each other but also with experimental data. The first one is the Simpson experiment, a classical water hammer test case and the second one is the Canon experiment consisting in the rapid depressurization of hot liquid water in a horizontal pipe. An important guideline of our work is that we focus on the comparison of the three models and not on the comparison of numerical schemes used for those models. Therefore, the following section also includes grid convergence studies in order to reduce the numerical approximations as far as possible.

\subsection{Simpson's column-separation water hammer}

The Simpson facility [40] consists of a $36 \mathrm{~m}$ long horizontal pipe linked to a tank and ending with a valve (see Fig. 3). Initially, a liquid water flow fills the pipe with a given velocity. At time zero, the valve is suddenly closed, thus generating a pressure wave that propagates towards the tank. Pressure is measured along the pipe at differents locations denoted P1, P2 and P3 in Figure 3. Due to the reflections at both ends of the pipe 


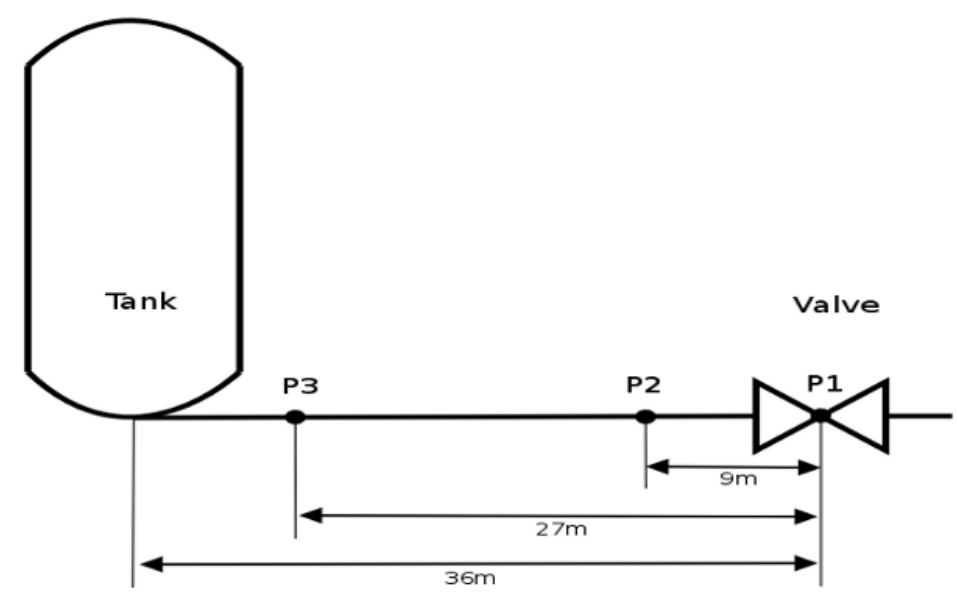

Figure 3. Schematic of Simpson test facility.

TABLE 5. Stiffened gas EOS parameters for Simpson experiment.

\begin{tabular}{cccccc}
\hline & $\gamma_{k}$ & $\begin{array}{c}p_{k}^{\infty} \\
(\mathrm{Pa})\end{array}$ & $\begin{array}{c}q_{k} \\
\left(\mathrm{~J} \mathrm{~kg}^{-1}\right)\end{array}$ & $\begin{array}{c}C_{V k} \\
\left(\mathrm{~J} \mathrm{~kg}^{-1} \mathrm{~K}^{-1}\right)\end{array}$ & $\begin{array}{c}q_{k}^{\prime} \\
\left(\mathrm{J} \mathrm{kg}^{-1} \mathrm{~K}^{-1}\right)\end{array}$ \\
\hline Vapor & 1.34 & 0.00 & 2009800.00 & 1344.06 & 1977.08 \\
Liquid & 2.27 & 692754002.87 & -1142331.00 & 1840.48 & 24218.87 \\
\hline
\end{tabular}

TABLE 6. Initial conditions for Simpson experiment.

\begin{tabular}{ccccc}
\hline & $\alpha_{k}$ & $\begin{array}{c}\rho_{k} \\
\left(\mathrm{~kg} \mathrm{~m}^{-3}\right)\end{array}$ & $\begin{array}{c}p_{k} \\
(\mathrm{bar})\end{array}$ & $\begin{array}{c}u_{k} \\
\left(\mathrm{~m} \mathrm{~s}^{-1}\right)\end{array}$ \\
\hline Vapor & $10^{-6}$ & 2.53 & 3.419 & 0.401 \\
Liquid & $1-10^{-6}$ & 997.90 & 3.419 & 0.401 \\
\hline
\end{tabular}

(the tank and the closed valve), different pressure peaks appear on the measurements. Moreover, if the initial velocity is high enough, pressure decreases towards the saturation pressure at the valve and a vapor pocket appears. This vapor pocket has a significant influence on the reflections of the pressure waves. Experimental measurements are compared to the results of 1D simulations of the two-fluid models.

An important conclusion of Simpson's work is that the influence of the elasticity of the pipe on the effective speed of sound must be taken into account in order to obtain a fair agreement between simulations and experimental data. Thus we use the Stiffened Gas EOS parameters presented in [11] which are chosen to recover the experimental speed of sound (see Tab. 5). The initial conditions used in the simulations are presented in Table 6. They are the same in the pipe and in the tank, and they correspond to the temperature of the experiment $T=296.3 \mathrm{~K}$ in both phases. The temperature is assumed to be constant in the simulation so we neglect the temperature relaxation: $\tau_{T}=+\infty$. The chemical relaxation time scale is set to $\tau_{\mu}=10^{-3} \mathrm{~s}$ with $\mu_{\text {ref }}=\left|\mu_{1}\right|+\left|\mu_{2}\right|$. We recall that $\tau_{p}$ and $\tau_{u}$ are respectively defined by equations (2.12) and (2.14).

First of all, different mesh refinements have been used in order to carry out a grid convergence study with the CGHS model. As it is shown in Figure 4, grid independance is obtained with 50000 cells: only a few differences on the second pressure peak (occuring around $300 \mathrm{~ms}$ ) can be observed between the two finest grids. Similar grid sensitivy has been observed for the BN models. Note that all results have been obtained with a CFL condition of 0.99 on 1D meshes thanks to the Europlexus fast transient dynamics software [15] where all the previous models and algorithms have been implemented. Then results for all models on the mesh made of 50000 cells 


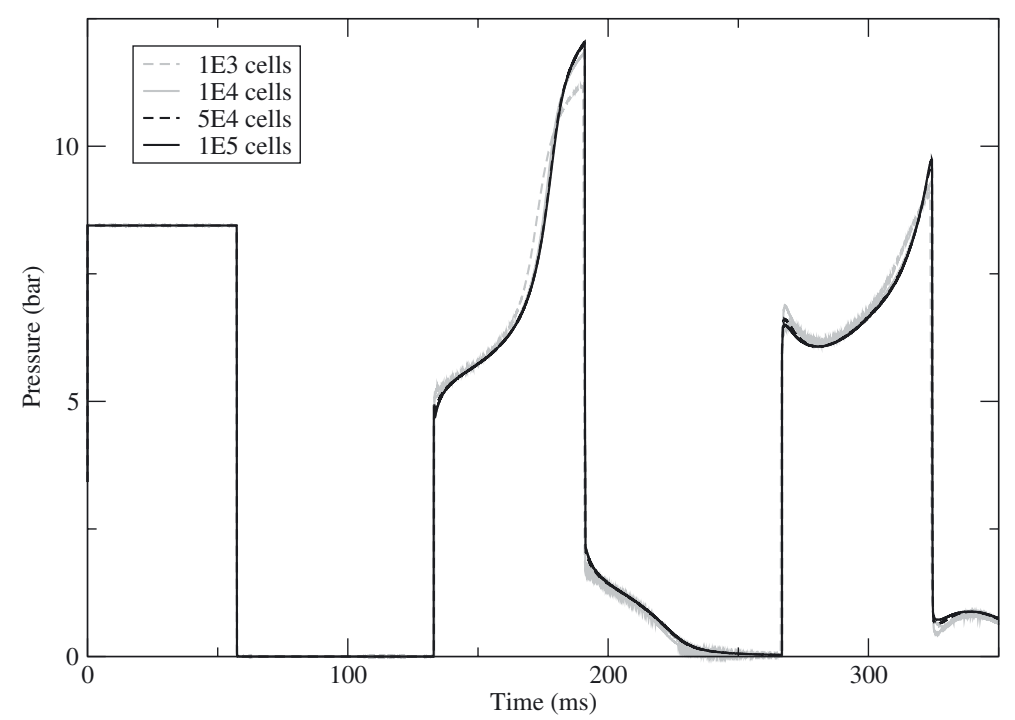

FIgURE 4. Influence of the grid refinement on the history of the mean pressure $p=\alpha_{l} p_{l}+\alpha_{v} p_{v}$ at P1 in Simpson water hammer experiment for CGHS model.

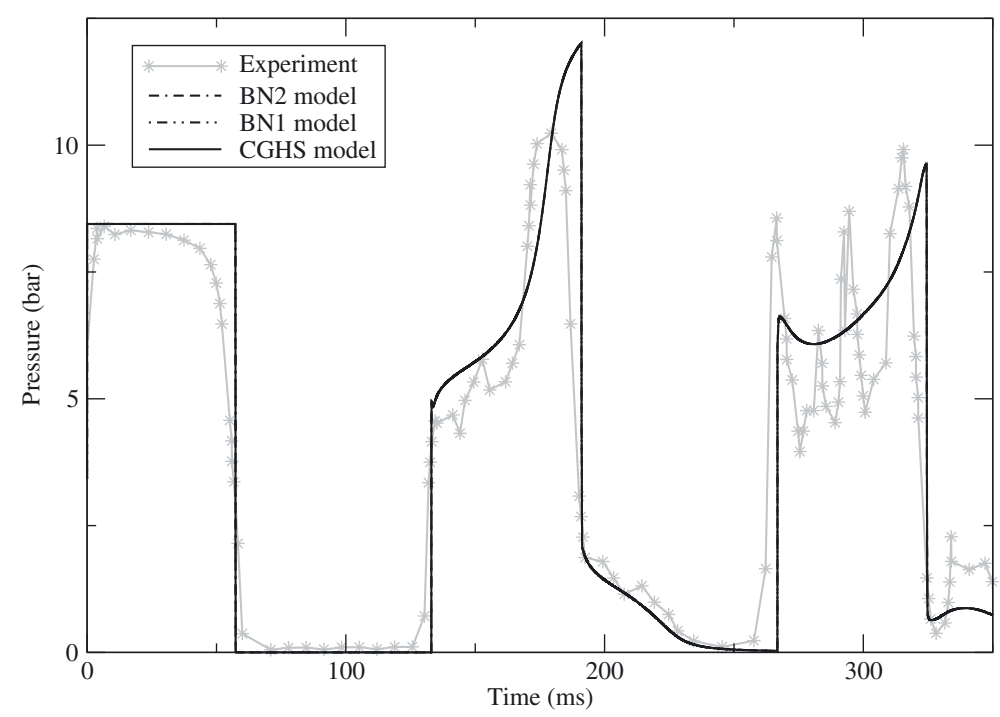

FiguRE 5. Mean pressure $p=\alpha_{l} p_{l}+\alpha_{v} p_{v} v s$. time at P1 in Simpson water hammer experiment: comparison between the CGHS model, the BN models and the experiment.

could be compared with the experimental results. Figure 5 provides us the comparison of the mean pressure $p=\alpha_{l} p_{l}+\alpha_{v} p_{v}$ history at the valve. We first notice that we are not able to distinguish the results of the different models. Close results are expected, especially with pressure and velocity relaxations, however the convective parts of the models are so different (see Fig. 2) that it is rather surprising. Then, we compare the numerical results with the experimental ones. If we focus on the pressure peak at $125 \mathrm{~ms}$, we have an excellent agreement on the times when pressure waves reach the valve. However the amplitude of the peak is a bit overestimated but we think that the addition of a wall friction model could drive it closer to the experimental results. 


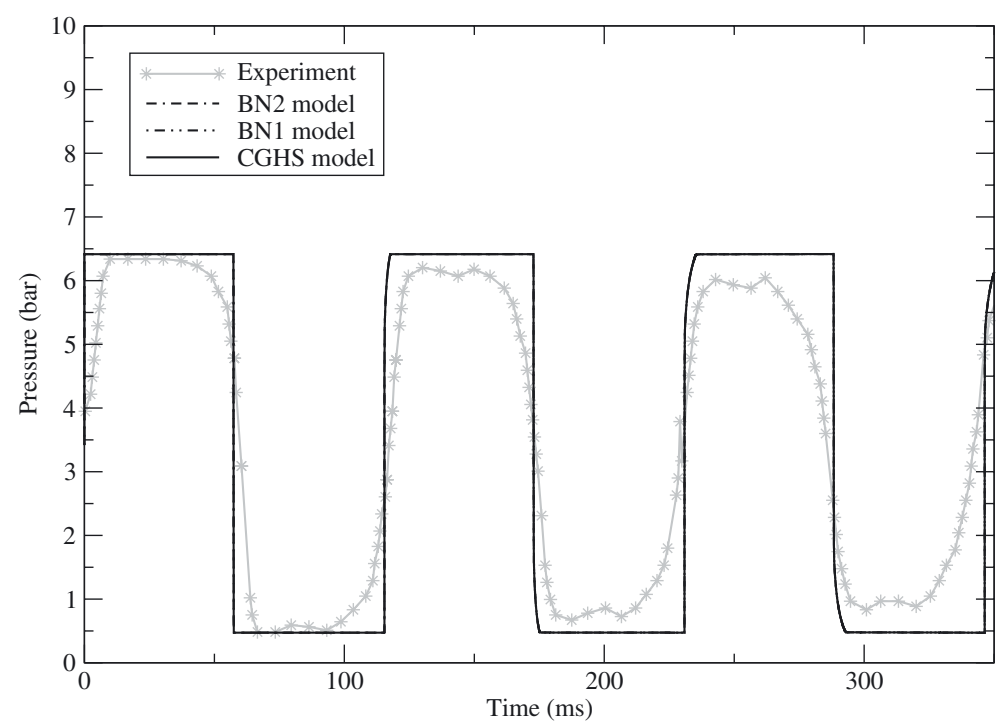

FiguRE 6. Mean pressure $p=\alpha_{l} p_{l}+\alpha_{v} p_{v} v s$. time at P1 in Simpson water hammer experiment with a smaller initial velocity $\left(u_{0}=0.239 \mathrm{~m} \mathrm{~s}^{-1}\right)$ : comparison between the CGHS model, the $\mathrm{BN}$ models and the experiment.

Simpson also provides experimental results with other initial velocities. For smaller initial velocities $\left(u_{0}=\right.$ $0.239 \mathrm{~m} \mathrm{~s}^{-1}$ for example), mass transfer no longer occurs so that the water hammer is purely monophasic. Obviously, the overall phenomenum is much easier to understand in this case, but it is also a good opportunity to highlight the lack of a wall friction model in our simulations. Figure 6 shows the comparison between the CGHS model, the BN models and the experiment in the monophasic case. As expected in a monophasic case, the results of the different models look the same. If we compare them to the experimental results, we see a very good agreement on the period of pressure peaks but their amplitudes are also slightly overestimated. It underlines the lack of physical dissipative phenomena in the present simulations.

\subsection{Discharge of a hot liquid from Canon experiment}

The Canon experiment [37] consists in the fast depressurization of water contained in a $4.389 \mathrm{~m}$ long pipe. The initial pressure and temperature of the water are high: 32 bar and $493.15 \mathrm{~K}$. At time zero, a membrane located at the end of the pipe is fully broken. It generates a rarefaction wave propagating along the pipe and the liquid water flashes. As detailed in Figure 7, several pressure transducers are disposed along the pipe (from P1 to P5) and a void fraction transducer is used at point Pt (between P2 and P3). Experimental results show that the liquid fully flashes therefore this is an excellent test case with vanishing phases. We emphasize the fact that the CGHS model makes more sense than the BN model in those cases from a theoretical point of view since the interfacial velocity $u_{I}$ of this model depends on the void fraction unlike the interfacial velocity of the BN models. The computations are still performed with the Europlexus fast transient dynamics software [15].

Computational domains used in Canon test case are similar to those used for Simpson test case: a 1D grid with a tank at one side and a closed end at the other. However, it should be emphasized that the initial conditions (see Tab. 8) are different in the tank and in the pipe unlike in Simpson experiment. Indeed, atmospheric conditions are applied in the tank in this case. Table 7 provides the Stiffened Gas EOS parameters. They are chosen to recover the water phase diagram as in [11]. Moreover the relaxation time scales $\tau_{p}$ and $\tau_{u}$ are respectively defined by equations (2.12) and (2.14). We also set $\tau_{T}=10^{-7} \mathrm{~s}$ and $\tau_{\mu}=5 \times 10^{-5} \mathrm{~s}$ with $\mu_{\text {ref }}=\left|\mu_{1}\right|+\left|\mu_{2}\right|$.

As in the previous experimental test case, a grid convergence study of the numerical results is carried out. Figure 8 shows the results for the CGHS model with a 0.9 CFL condition: beyond 5000 cells, results are no 


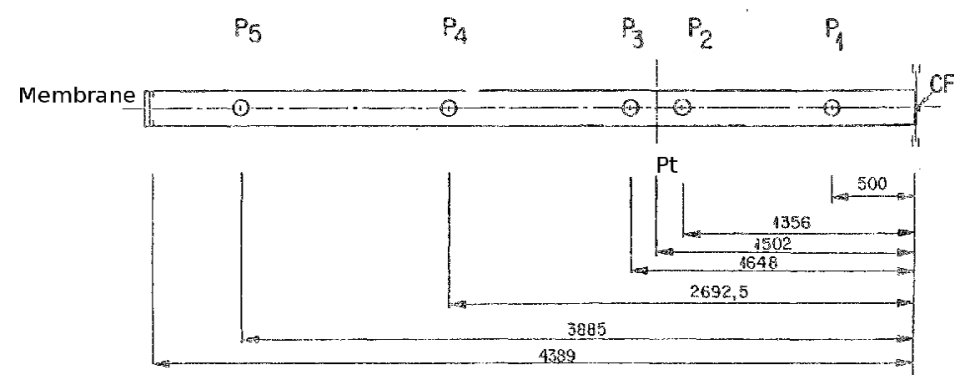

FiguRE 7. Schematic of Canon test facility.

TABLE 7. EOS parameters for Canon experiment.

\begin{tabular}{lccccc}
\hline & $\gamma_{k}$ & $\begin{array}{c}p_{k}^{\infty} \\
(\mathrm{Pa})\end{array}$ & $\begin{array}{c}q_{k} \\
\left(\mathrm{~J} \mathrm{~kg}^{-1}\right)\end{array}$ & $\begin{array}{c}C_{V k} \\
\left(\mathrm{~J} \mathrm{~kg}^{-1} \mathrm{~K}^{-1}\right)\end{array}$ & $\begin{array}{c}q_{k}^{\prime} \\
\left(\mathrm{J} \mathrm{kg}^{-1} \mathrm{~K}^{-1}\right)\end{array}$ \\
\hline Vapor & 1.34 & 0.00 & 2032350.00 & 1162.00 & 2351.11 \\
Liquid & 1.66 & 769317123.86 & -1359570.00 & 2807.61 & 11671.61 \\
\hline
\end{tabular}

TABLE 8. Initial conditions for Canon experiment.

\begin{tabular}{cccccc}
\hline & & $\alpha_{k}$ & $\begin{array}{c}\rho_{k} \\
\left(\mathrm{~kg} \mathrm{~m}^{-3}\right)\end{array}$ & $\begin{array}{c}p_{k} \\
(\mathrm{bar})\end{array}$ & $\begin{array}{c}u_{k} \\
\left(\mathrm{~m} \mathrm{~s}^{-1}\right)\end{array}$ \\
\hline \multirow{2}{*}{ Pipe } & Vapor & $10^{-3}$ & 16.72 & 32 & 0.00 \\
& Liquid & $1-10^{-3}$ & 841.12 & 32 & 0.00 \\
\hline \multirow{2}{*}{ Tank } & Vapor & $1-10^{-3}$ & 0.52 & 1 & - \\
& Liquid & $10^{-3}$ & 837.74 & 1 & - \\
\hline
\end{tabular}

longer sensitive to the mesh refinement. Thus results on the finest grid (10000 cells) are then compared to the experimental data. As it is shown in Figures 9 and 10, numerical results of the different models are also completely similar in this case. Like the experimental data, numerical models show the sudden pressure drop, then the pressure remains constant around the saturation pressure value during the vaporization and slowly decreases to reach the atmospheric pressure. The main difference between the numerical simulations and the experimental results is that the vaporization process is predicted earlier in the simulations but apart from that a good agreement is observed.

Although pressure and velocity relaxations are expected to lead to similar results for all models, the fact that no visible difference can be observed on the numerical results of this test case with vanishing phases is surprising. In order to investigate in more depth the effect of the relaxation terms, Figure 11 shows normalized pressure and velocity differences between phases over time at $\mathrm{Pt}\left(p_{0}=32\right.$ bar is the initial pressure in the pipe and $c_{0}=1234 \mathrm{~m} \mathrm{~s}^{-1}$ the celerity of pressure waves in liquid water at $32 \mathrm{bar}$ ). Thus, we observe that the normalized pressure and velocity differences are respectively lower than $1 \%$ and $3 \%$. We can also evaluate the order of magnitude of both $\tau_{p}$ and $\tau_{u}$. If we assume that $p_{\text {ref }}=1$ bar and the dynamic viscosities are $\eta_{1}=10^{-5} \mathrm{~kg} \mathrm{~m}^{-1} \mathrm{~s}^{-1}$ and $\eta_{2}=10^{-4} \mathrm{~kg} \mathrm{~m}^{-1} \mathrm{~s}^{-1}$, equation (2.12) gives:

$$
10^{-10} \mathrm{~s} \leq \tau_{p} \leq 10^{-9} \mathrm{~s} .
$$

If we also assume that the surface tension $\sigma=5 \times 10^{-2} \mathrm{~N} \mathrm{~m}^{-1}$, the densities $\rho_{1}=20 \mathrm{~kg} \mathrm{~m}^{-3}, \rho_{2}=800 \mathrm{~kg} \mathrm{~m}^{-3}$ and the velocity difference $\left|u_{1}-u_{2}\right|=10 \mathrm{~m} \mathrm{~s}^{-1}$ (thanks to Fig. 11), equation (2.14) gives:

$$
6 \times 10^{-9} \mathrm{~s} \leq \tau_{u} \leq 8 \times 10^{-4} \mathrm{~s} .
$$




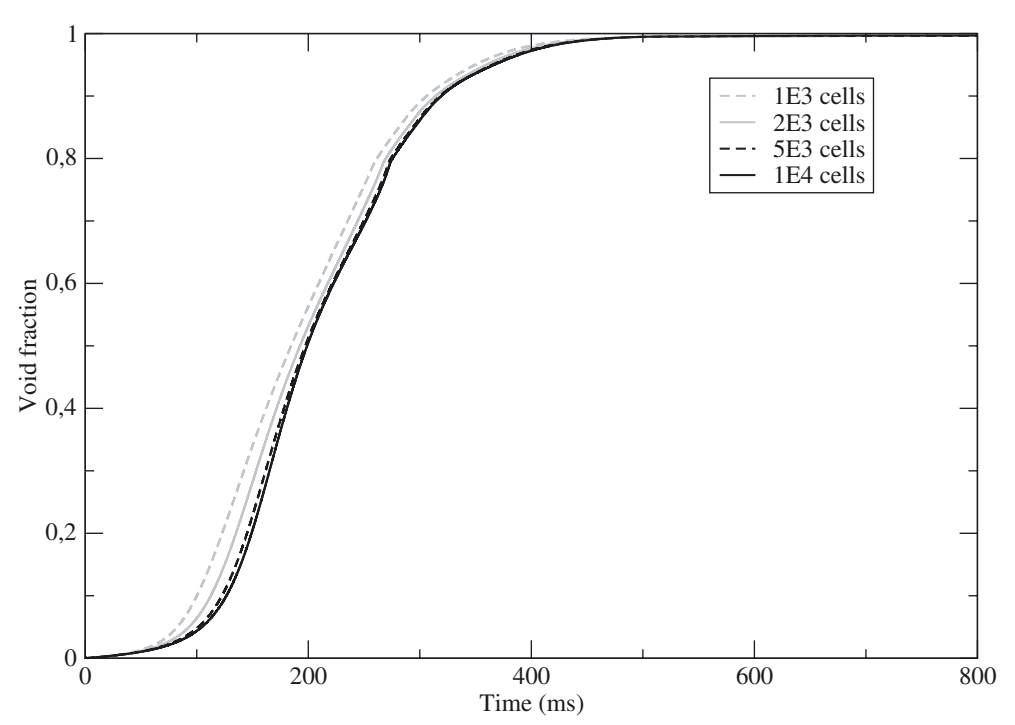

Figure 8. Influence of the grid refinement on the history of the void fraction at Pt in Canon experiment for CGHS model.

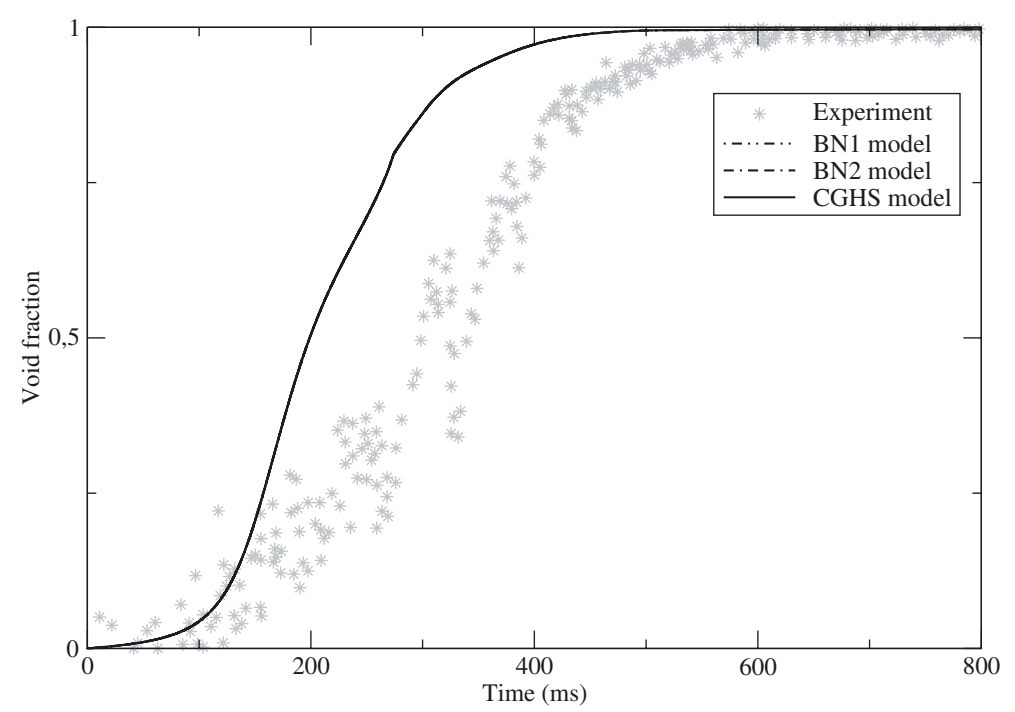

FIgURE 9. Void fraction vs. time at Pt in Canon experiment: comparison between the CGHS model, the BN models and the experiment.

We can notice that unlike $\tau_{p}$, the order of magnitude of $\tau_{u}$ strongly depends on the void fraction because of the difference of densities between the two phases. Both those relaxation time scales need to be compared with the time step given by the CFL condition which is $\Delta t \approx 3 \times 10^{-7} \mathrm{~s}$ initially. Therefore, $\tau_{p}$ is small compared to the time step and it leads to the small pressure difference which can be observed in Figure 11. In the case of $\tau_{u}$, the effect of the relaxation during the transient is less clear since $\tau_{u}$ can become greater than the time step when the flow is mainly made of vapor. This can be observed in Figure 11 after $300 \mathrm{~ms}$, at the end of the vaporization, $\tau_{u}$ grows and so does the velocity difference. However, it does not exceed $3 \%$ as pointed out before. On the whole, pressure and velocity differences remain small due to small relaxation time scales hence 


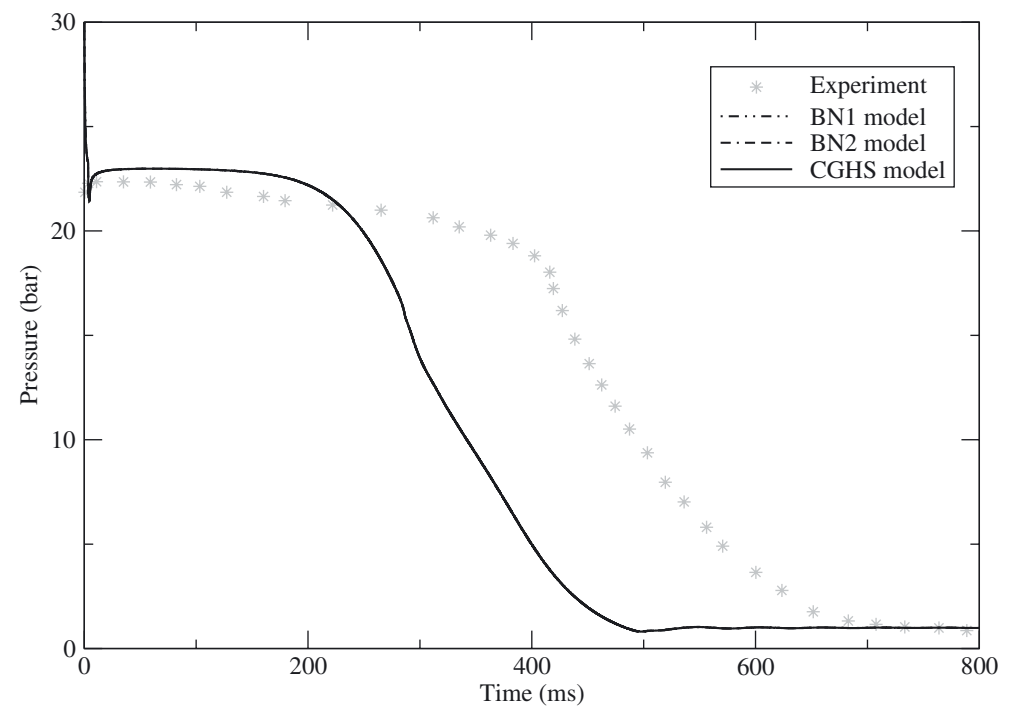

Figure 10. Mean pressure $p=\alpha_{l} p_{l}+\alpha_{v} p_{v}$ vs. time at Pt in Canon experiment: comparison between the CGHS model, the BN models and the experiment.
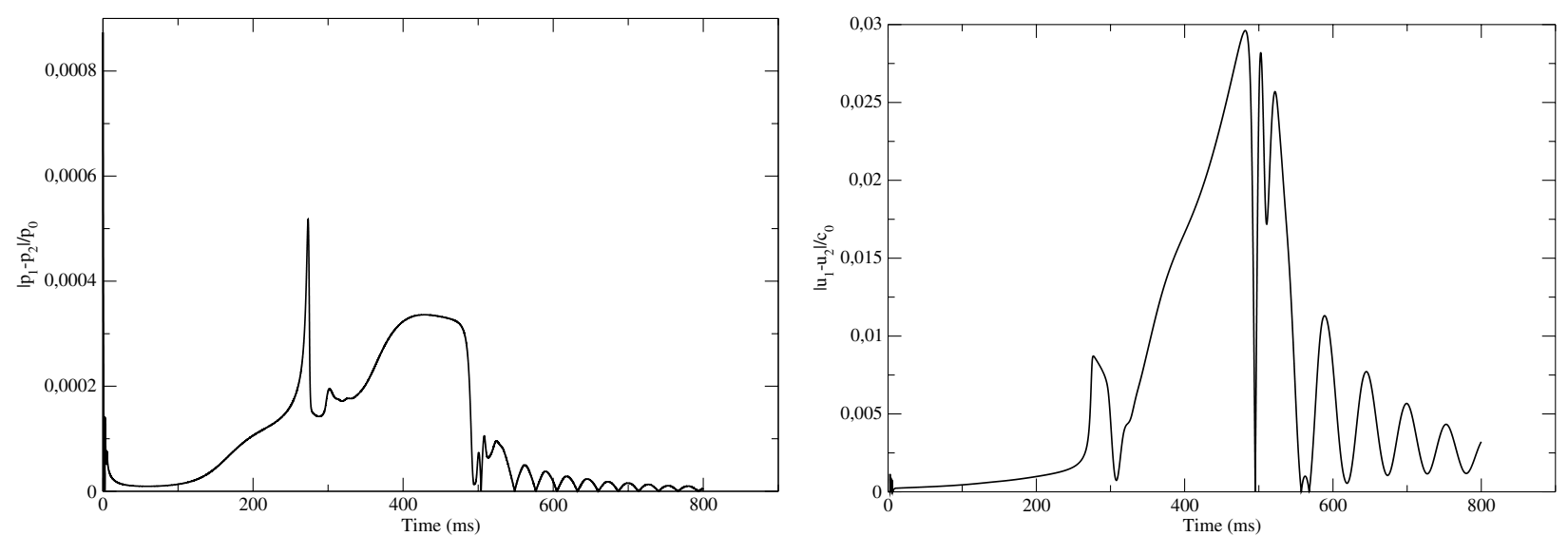

Figure 11. Normalized pressure (left, $p_{0}=32$ bar) and velocity (right, $c_{0}=1234 \mathrm{~m} \mathrm{~s}^{-1}$ ) differences between phases vs. time at $\mathrm{Pt}$ in Canon experiment.

the numerical results of the different models are similar on this test case. Unfortunately, this result can not easily be extended to other applications, even other steam-water applications, due to the strong dependance of $\tau_{u}$ on the void fraction. Indeed the mass transfer, which is a complex phenomenum in steam-water flows, has a significant effect on the void fraction and thus on the relaxation effects.

\section{CONCLUSion}

Different two-phase flow models have been presented and compared with each other in steam-water transient applications. They all are two-fluid models and they exhibit the same mathematical properties. Thus, similar approaches have been used to compute numerical approximations of those models. It is based on a fractional step method that complies with the key property of all models, an overall entropy inequality. For each step 
and substep of the method, rather simple numerical schemes have been used and particular attention have been paid to their verification with grid convergence studies. We emphasize this point because it is of major importance if we want a comparison between models with only a few numerical approximations. We also recall that schemes for relaxation terms proposed in [24] have been extended to Stiffened Gas EOS and that pressure relaxation in the case of the CGHS model has required the proposition of a new scheme.

From a theoretical point of view, pressure and velocity relaxation phenomena are expected to drive models closer to each other. Therefore we require physically relevant implementations of those two substeps and of the corresponding approximations of the relaxation time scales. For both test cases involving steam-water transients with mass transfer, the three models give a fair agreement with experimental data. An important conclusion of the present work is that all three models provide almost the same converged numerical results on those test cases, even with vanishing phases. It is surprising regarding the differences between the convective part of the models. Further work should be devoted to confirm this trend and also to improve the modelling of the mass transfer in order to obtain numerical results closer to the experimental data. The chemical potential relaxation time scale seems to be a key parameter in the current models, however only little attention has been paid to this for non-homogeneous models in the literature.

\section{Appendix A. A Class of TWO-Fluid MODElS}

The new framework proposed in [23] considers the scalar $\chi$ which is introduced in the closure law of the interfacial velocity $u_{I}$ as a new variable of a class of two-fluid models. This variable is a non-dimensional scalar lying in $[0,1]$ that characterizes the flow regime. The corresponding set of equations reads:

$$
\left\{\begin{array}{lll}
\partial_{t}(\chi) & +\omega \partial_{x}(\chi) & =\chi(\chi-1)\left(\chi-\frac{1}{2}\right) S_{\chi} \\
\partial_{t}\left(\alpha_{k}\right)+u_{I} \partial_{x}\left(\alpha_{k}\right) & =S_{1, k} \\
\partial_{t}\left(\alpha_{k} \rho_{k}\right)+\partial_{x}\left(\alpha_{k} \rho_{k} u_{k}\right) & =S_{2, k} \\
\partial_{t}\left(\alpha_{k} \rho_{k} u_{k}\right)+\partial_{x}\left(\alpha_{k} \rho_{k} u_{k}^{2}+\alpha_{k} p_{k}\right)-p_{I} \partial_{x}\left(\alpha_{k}\right) & =S_{3, k} \\
\partial_{t}\left(\alpha_{k} \rho_{k} e_{k}\right)+\partial_{x}\left(\alpha_{k} \rho_{k} e_{k} u_{k}+\alpha_{k} p_{k} u_{k}\right)-p_{I} u_{I} \partial_{x}\left(\alpha_{k}\right) & =S_{4, k}
\end{array}\right.
$$

The same closure laws as in Section 2.1 are used:

$$
\begin{array}{ll}
u_{I}=a u_{1}+(1-a) u_{2} & a=\frac{\chi m_{1}}{\chi m_{1}+(1-\chi) m_{2}}, \\
p_{I}=b p_{1}+(1-b) p_{2} & b=\frac{(1-a) T_{2}}{a T_{1}+(1-a) T_{2}},
\end{array}
$$

and the source terms $S_{j, k}$ are defined by equations (2.5) and (2.6). The velocity $\omega$ is closed so that the field associated with $\lambda=\omega$ is linearly degenerate. Two different approaches are proposed in [23]:

$$
\omega=\frac{m_{1} u_{1}+m_{2} u_{2}}{m_{1}+m_{2}} \quad \text { or } \quad \omega=0 .
$$

The first choice ensures that the corresponding field is linearly degenerate and follows a moving interface between two domains. The second choice is rather equivalent to user-defined domains where particular models are used, depending on the dominant phase for example. In that case, it is important to note that the BN and CGHS models $\left(\chi=0, \frac{1}{2}, 1\right)$ provide particular solutions of the first equation of system (A.1). We briefly recall the properties of this class of models. Details and proofs can be found in [23]. 


\section{- Entropy inequality}

Smooth solutions of system (A.1) with the previous closure laws comply with an entropy inequality:

$$
\partial_{t}\left(\sum_{k} m_{k} s_{k}\right)+\partial_{x}\left(\sum_{k} m_{k} s_{k} u_{k}\right) \geq 0 .
$$

\section{- Hyperbolicity and structure of waves}

The convective part of system (A.1) is hyperbolic and admits eight real eigenvalues:

$$
\begin{gathered}
\lambda_{1}=\omega, \quad \lambda_{2}=u_{I}, \\
\lambda_{3}=u_{1}-c_{1}, \quad \lambda_{4}=u_{1}, \quad \lambda_{5}=u_{1}+c_{1}, \\
\lambda_{6}=u_{2}-c_{2}, \quad \lambda_{7}=u_{2}, \quad \lambda_{8}=u_{2}+c_{2} .
\end{gathered}
$$

Associated righteigenvectors span the whole space $\mathbb{R}^{8}$ if $\left|u_{k}-u_{I}\right| \neq c_{k}$ and $\left|u_{k}-\omega\right| \neq c_{k}$. Fields associated with eigenvalues $\lambda_{1,2,4,7}$ are linearly degenerate (LD) whereas fields associated with eigenvalues $\lambda_{3,5,6,8}$ are genuinely non linear (GNL).

\section{- Jump conditions}

Unique jump conditions hold within each isolated field. Moreover, classical single phase jump relations hold in the GNL fields:

$$
\left\{\begin{array}{c}
{[\chi]=0,} \\
{\left[\alpha_{k}\right]=0,} \\
-\sigma\left[\rho_{k}\right]+\left[\rho_{k} u_{k}\right]=0, \\
-\sigma\left[\rho_{k} u_{k}\right]+\left[\rho_{k} u_{k}^{2}+p_{k}\right]=0, \\
-\sigma\left[\rho_{k} e_{k}\right]+\left[\rho_{k} e_{k} u_{k}+p_{k} u_{k}\right]=0 .
\end{array}\right.
$$

\section{Appendix B. Verification of the pressure Relaxation scheme}

This appendix is dedicated to the verification of the pressure relaxation scheme presented in Section 3.2.1. In the case of BN models, it can be found in [24] so we focus on the case of the CGHS model. Thus, we recall that approximate solutions of the following system:

$$
\begin{cases}\partial_{t}\left(\alpha_{k}\right) & =\Phi_{k} \\ \partial_{t}\left(\alpha_{k} \rho_{k}\right) & =0 \\ \partial_{t}\left(\alpha_{k} \rho_{k} u_{k}\right) & =0 \\ \partial_{t}\left(\alpha_{k} \rho_{k} e_{k}\right) & =-p_{I} \Phi_{k}\end{cases}
$$

are computed using the following scheme:

$$
\left\{\begin{aligned}
\frac{\alpha_{k}^{*}-\alpha_{k}^{0}}{\Delta t} & =\frac{1}{\left(\tau_{p} p_{\text {ref }}\right)^{0}} \alpha_{k}^{*} \alpha_{j}^{*}\left(p_{k}^{*}-p_{j}^{*}\right), \\
\frac{m_{k}^{*} \varepsilon_{k}^{*}-m_{k}^{0} \varepsilon_{k}^{0}}{\Delta t} & =-\left(b^{0} p_{1}^{*}+\left(1-b^{0}\right) p_{2}^{*}\right) \frac{\alpha_{k}^{*}-\alpha_{k}^{0}}{\Delta t}, \quad j=3-k
\end{aligned}\right.
$$

which is equivalent to a non-linear system in $\alpha_{1}^{*}, p_{1}^{*}$ and $p_{2}^{*}$. The parameters of the Stiffened Gas used in the verification test case (see Tab. B.1) are chosen so that an analytical solution of system (B.1) could be provided. The initial conditions of the test case are presented in Table B.2 and the pressure relaxation time scale is set in the following way: $\tau_{p} p_{\text {ref }}=\frac{4}{3} \mu$ with $\mu=10^{-3} \mathrm{~kg} \mathrm{~m}^{-1} \mathrm{~s}^{-1}$. Figure B.1 shows that a first-order of convergence is retrieved, as expected. 
TABLE B.1. EOS parameters for the pressure relaxation test case.

\begin{tabular}{cccccc}
\hline & $\gamma_{k}$ & $\begin{array}{c}p_{k}^{\infty} \\
(\mathrm{en} \mathrm{Pa})\end{array}$ & $\begin{array}{c}q_{k} \\
\left(\mathrm{~J} \mathrm{~kg}^{-1}\right)\end{array}$ & $\begin{array}{c}C_{V k} \\
\left(\mathrm{~J} \mathrm{~kg}^{-1} \mathrm{~K}^{-1}\right)\end{array}$ & $\begin{array}{c}q_{k}^{\prime} \\
\left(\mathrm{J} \mathrm{kg}^{-1} \mathrm{~K}^{-1}\right)\end{array}$ \\
\hline Phase 1 & 2 & 0.00 & 0.00 & 1500 & 0 \\
Phase 2 & 2 & 0.00 & 0.00 & 1500 & 0 \\
\hline
\end{tabular}

TABLE B.2. Initial conditions for the pressure relaxation test case.

\begin{tabular}{ccccc}
\hline & $\alpha_{k}$ & $\begin{array}{c}\rho_{k} \\
\left(\mathrm{~kg} \mathrm{~m}^{-3}\right)\end{array}$ & $\begin{array}{c}p_{k} \\
(\mathrm{~Pa})\end{array}$ & $\begin{array}{c}u_{k} \\
\left(\mathrm{~m} \mathrm{~s}^{-1}\right)\end{array}$ \\
\hline Phase 1 & 0.8 & 2 & 100000 & 50 \\
Phase 2 & 0.2 & 1000 & 1600000 & -20 \\
\hline
\end{tabular}

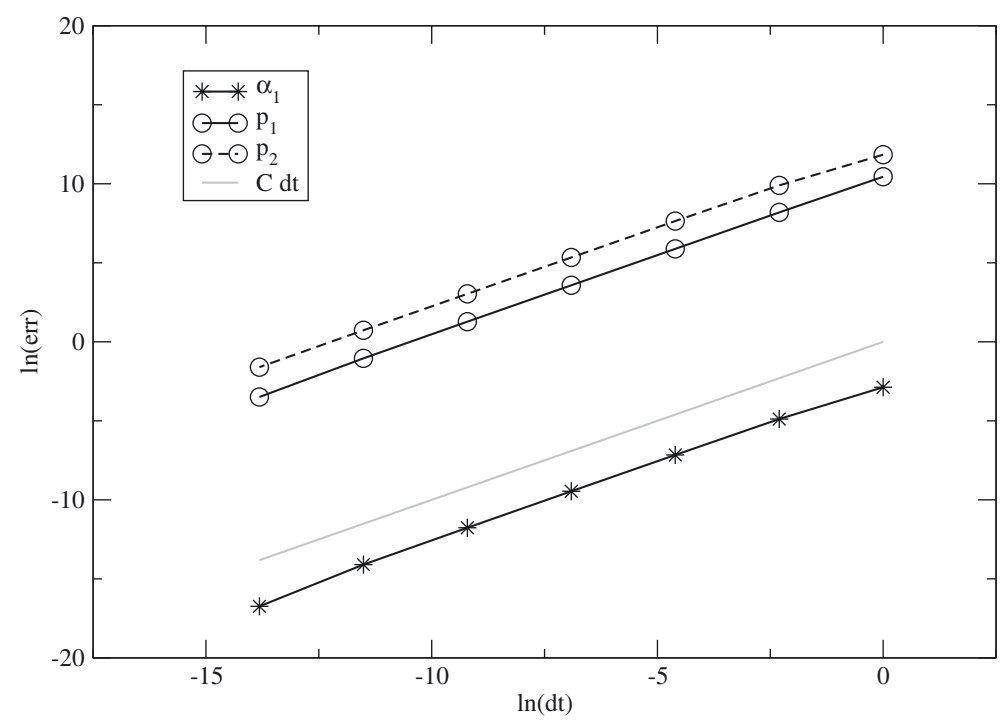

Figure B.1. Convergence study of the pressure relaxation scheme: $L 1$ norm of the error $v s$. $\Delta t$ at $t_{f}=10^{-9} \mathrm{~s}$.

\section{Appendix C. Verification of the Chemical potential Relaxation scheme}

This appendix is dedicated to the verification of the chemical potential relaxation scheme presented in Section 3.2.4. Approximate solutions of the following system are computed:

$$
\left\{\begin{array}{l}
\partial_{t}\left(\alpha_{k}\right)=0 \\
\partial_{t}\left(m_{k}\right)=\Gamma_{k}, \\
\partial_{t}\left(m_{k} u_{k}\right)=\mathcal{U} \Gamma_{k}, \\
\partial_{t}\left(m_{k} \varepsilon_{k}\right)=0
\end{array}\right.
$$


To that end, an implicit scheme is used. First, $m_{k}^{*}$ is determined by solving the following non-linear equations:

$$
\frac{m_{k}^{*}-m_{k}^{0}}{\Delta t}=\frac{1}{\left(\tau_{\mu} \mu_{\mathrm{ref}}\right)^{0}} \frac{m_{k}^{*} m_{j}^{*}}{m_{k}^{*}+m_{j}^{*}}\left(\mu_{j}^{*}-\mu_{k}^{*}\right), \quad j=3-k .
$$

Then, $u_{k}^{*}$ can be easily computed as the solution of a linear system:

$$
\frac{m_{k}^{*} u_{k}^{*}-m_{k}^{0} u_{k}^{0}}{\Delta t}=\frac{u_{k}^{*}+u_{j}^{*}}{2} \frac{m_{k}^{*}-m_{k}^{0}}{\Delta t}, \quad j=3-k .
$$

As in the case of pressure relaxation, we provide a verification test case with a particular choice of the EOS parameters (Tab. C.1) in order to have an analytical solution of system (C.1). Table C.2 gathers the initial conditions of the test case. The chemical potential relaxation time scale is set to $\tau_{\mu}=10^{-3} \mathrm{~s}$ and the reference chemical potential to $\mu_{\mathrm{ref}}=10^{4} \mathrm{~J} \mathrm{~kg}^{-1} \mathrm{~K}^{-1}$. As we can see in Figure C.1, we retrieve the expected first order of convergence.

TABLE C.1. EOS parameters for the chemical potential relaxation test case.

\begin{tabular}{cccccc}
\hline & $\gamma_{k}$ & $\begin{array}{c}p_{k}^{\infty} \\
(\mathrm{Pa})\end{array}$ & $\begin{array}{c}q_{k} \\
\left(\mathrm{~J} \mathrm{~kg}^{-1}\right)\end{array}$ & $\begin{array}{c}C_{V k} \\
\left(\mathrm{~J} \mathrm{~kg}^{-1} \mathrm{~K}^{-1}\right)\end{array}$ & $\begin{array}{c}q_{k}^{\prime} \\
\left(\mathrm{J} \mathrm{kg}^{-1} \mathrm{~K}^{-1}\right)\end{array}$ \\
\hline Phase 1 & 1.4 & 100000 & 0 & 3125 & 2000 \\
Phase 2 & 2.5 & 7000000 & 0 & 1750 & 25000 \\
\hline
\end{tabular}

TABLE C.2. Initial conditions for the chemical potential relaxation test case.

\begin{tabular}{ccccc}
\hline & $\alpha_{k}$ & $\begin{array}{c}\rho_{k} \\
\left(\mathrm{~kg} \mathrm{~m}^{-3}\right)\end{array}$ & $\begin{array}{c}p_{k} \\
(\mathrm{~Pa})\end{array}$ & $\begin{array}{c}u_{k} \\
\left(\mathrm{~m} \mathrm{~s}^{-1}\right)\end{array}$ \\
\hline Phase 1 & 0.2 & 2.5 & 2800000 & 50 \\
Phase 2 & 0.8 & 995 & 3400000 & 20 \\
\hline
\end{tabular}

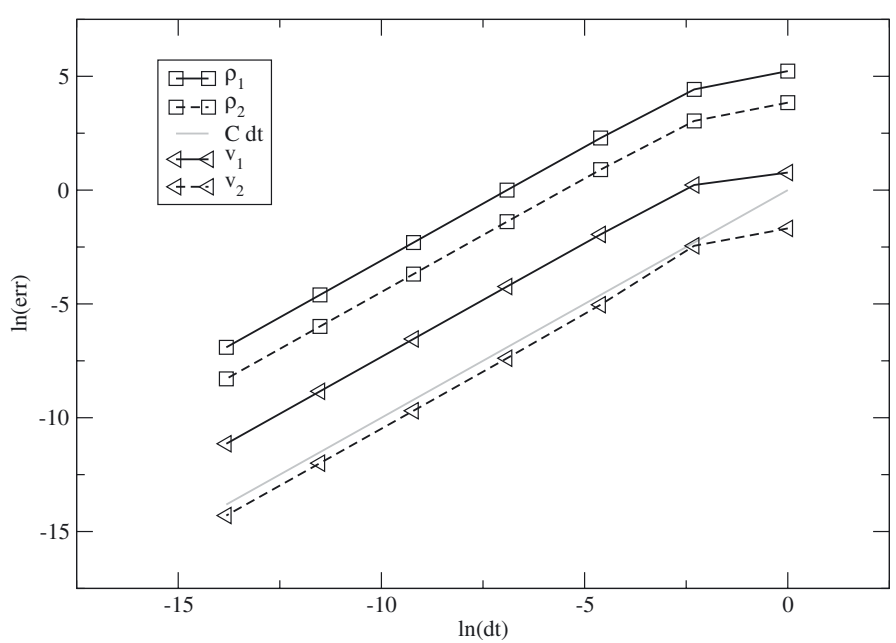

Figure C.1. Convergence study of the chemical potential relaxation scheme: $L 1$ norm of the error vs. $\Delta t$ at $t_{f}=5 \times 10^{-3} \mathrm{~s}$. 
Acknowledgements. The first author received a financial support through an EDF-CIFRE Contract 0459-2013. Computational facilities were provided by EDF. Numerical simulations have been performed with the Europlexus code.

\section{REFERENCES}

[1] A. Ambroso, C. Chalons and P.-A. Raviart, A Godunov-type method for the seven-equation model of compressible two-phase flow. Comput. Fluids 54 (2012) 67-91.

[2] N. Andrianov and G. Warnecke, The Riemann problem for the Baer-Nunziato two-phase flow model. J. Comput. Phys. 195 (2004) 434-464.

[3] M.R. Baer and J.W. Nunziato, A two-phase mixture theory for the deflagration-to-detonation transition (DDT) in reactive granular materials. Int. J. Multiphase Flow 12 (1986) 861-889.

[4] J.B. Bdzil, R. Menikoff, S.F. Son, A.K. Kapila and D.S. Stewart, Two-phase modeling of deflagration-to-detonation transition in granular materials: A critical examination of modeling issues. Phys. Fluids 11 (1999) 378-402.

[5] R. Berry, L. Zou, H. Zhao, D. Andrs, J. Peterson, H. Zhang and R. Martineau, Relap-7: Demonstrating Seven-Equation, TwoPhase Flow Simulation in a Single-Pipe, Two-Phase Reactor Core and Steam Separator/Dryer, Technical Report INL/EXT13-28750, Idaho National Laboratory (INL) (2013).

[6] A. Chinnayya, E. Daniel and R. Saurel, Modelling detonation waves in heterogeneous energetic materials. J. Comput. Phys. 196 (2004) 490-538.

[7] F. Coquel, T. Gallouët, J.-M. Hérard and N. Seguin, Closure laws for a two-fluid two-pressure model. C. R. Math. 334 (2002) 927-932.

[8] F. Coquel, J.-M. Hérard, K. Saleh and N. Seguin, A robust entropy-satisfying finite volume scheme for the isentropic BaerNunziato model. ESAIM: M2AN 48 (2014) 165-206.

[9] F. Crouzet, F. Daude, P. Galon, P. Helluy, J.-M. Hérard, O. Hurisse and Y. Liu, Approximate solutions of the Baer-Nunziato Model. ESAIM: Procs. 40 (2013) 63-82.

[10] F. Crouzet, F. Daude, P. Galon, J.-M. Hérard, O. Hurisse and Y. Liu, Validation of a two-fluid model on unsteady liquid-vapor water flows. Comput. Fluids 119 (2015) 131-142.

[11] F. Daude, P. Galon, Z. Gao and E. Blaud, Numerical experiments using a HLLC-type scheme with ALE formulation for compressible two-phase flows five-equation models with phase transition. Comput. Fluids 94 (2014) 112-138.

[12] V. Deledicque and M.V. Papalexandris, An exact Riemann solver for compressible two-phase flow models containing nonconservative products. J. Comput. Phys. 222 (2007) 217-245.

[13] P. Downar-Zapolski, Z. Bilicki, L. Bolle and J. Franco, The non-equilibrium relaxation model for one-dimensional flashing liquid flow. Int. J. Multiphase Flow 22 (1996) 473-483.

[14] D.A. Drew and S.L. Passman, Theory of Multicomponent Fluids. Springer Verlag (1999).

[15] Europlexus, User's Manual. Technical Report, Joint Reasearch Center (JRC), Commissariat à l'énergie atomique et aux énergies alternatives (CEA). Available at http://europlexus.jrc.ec.europa.eu/public/manual_html/index.html (2015).

[16] T. Gallouët, J.-M. Hérard and N. Seguin, Numerical modeling of two-phase flows using the two-fluid two-pressure approach. Math. Models Methods Appl. Sci. 14 (2004) 663-700.

[17] T. Gallouët, P. Helluy, J.-M. Hérard and J. Nussbaum, Hyperbolic relaxation models for granular flows. ESAIM: M2AN 44 (2010) 371-400.

[18] S. Gavrilyuk, The structure of pressure relaxation terms: one-velocity case. Internal report H-I83-2014-00276-EN, EDF R\&D (2014).

[19] S. Gavrilyuk and R. Saurel, Mathematical and numerical modeling of two-phase compressible flows with micro-inertia. J. Comput. Phys. 175 (2002) 326-360.

[20] J. Glimm, D. Saltz and D.H. Sharp, Renormalization group solution of two-phase flow equations for Rayleigh-Taylor mixing. Phys. Lett. A 222 (1996) 171-176.

[21] V. Guillemaud, Modélisation et simulation numérique des écoulements diphasiques par une approche bifluide à deux pressions. Ph.D. thesis, Université de Provence - Aix-Marseille I. Available at https://tel.archives-ouvertes.fr/tel-00169178/ document (2007).

[22] J.-M. Hérard, A three-phase flow model. Math. Comput. Model. 45 (2007) 732-755.

[23] J.-M. Hérard, Une classe de modèles diphasiques bifluides avec changement de régime. Internal report H-I81-2010-00486-FR, $\operatorname{EDF}(2010)$.

[24] J.-M. Hérard and O. Hurisse, A fractional step method to compute a class of compressible gas-liquid flows. Comput. Fluids 55 (2012) 57-69.

[25] J.-M. Hérard and Y. Liu, Une approche bifluide statistique de modélisation des écoulements diphasiques à phases compressibles. Internal report H-I81-2013-01162-FR, EDF (2013).

[26] M. Ishii, Thermo-fluid dynamic theory of two-phase flows. Collection de la Direction des Etudes et Recherches d'Electricité de France. Eyrolles, Paris (1975).

[27] H. Jin, J. Glimm and D.H. Sharp, Entropy of averaging for compressible two-pressure two-phase flow models. Phys. Lett. A 360 (2006) 114-121.

[28] A.K. Kapila, S.F. Son, J.B. Bdzil, R. Menikoff and D.S. Stewart, Two-phase modeling of DDT: Structure of the velocityrelaxation zone. Phys. Fluids 9 (1997) 3885-3897. 
[29] J. Laviéville, M. Boucker, E. Quemerais, S. Mimouni and N. Méchitoua, NEPTUNE_CFD V1.0 - Theory Manual. Internal report H-I81-2006-04377-EN. EDF R\&D (2006).

[30] O. Le Métayer, J. Massoni and R. Saurel, Élaboration des lois d'état d'un liquide et de sa vapeur pour les modèles d'écoulements diphasiques. Int. J. Thermal Sci. 43 (2004) 265-276.

[31] O. Le Métayer, J. Massoni and R. Saurel, Dynamic relaxation processes in compressible multiphase flows. Application to evaporation phenomena. ESAIM: Procs. 40 (2013) 103-123.

[32] H. Lochon, Modélisation d'écoulements diphasiques: fermetures entropiques de modèles bifluides. Internal report H-T63-201410406-FR. EDF (2014).

[33] S. Müller, M. Hantke, and P. Richter. Closure conditions for non-equilibrium multi-component models. Contin. Mech. Thermodyn. 28 (2016) 1157-1189.

[34] M. Pelanti and K.-M. Shyue, A mixture-energy-consistent six-equation two-phase numerical model for fluids with interfaces, cavitation and evaporation waves. J. Comput. Phys. 259 (2014) 331-357.

[35] V.H. Ransom and D.L. Hicks, Hyperbolic two-pressure models for two-phase flow. J. Comput. Phys. 53 (1984) $124-151$.

[36] RELAP5-3d, Code Manual Volume IV: Models and Correlations. Technical Report INEEL-EXT-98-00834, Idaho National Laboratory (INL) (2012).

[37] B. Riegel, Contribution à l'étude de la décompression d'une capacité en regime diphasique. Ph.D. thesis, Université Scientifique et Médicale et Institut National Polytechnique de Grenoble (1978).

[38] R. Saurel and R. Abgrall, A Multiphase Godunov Method for Compressible Multifluid and Multiphase Flows. J. Comput. Phys. 150 (1999) 425-467.

[39] D.W. Schwendeman, C.W. Wahle and A.K. Kapila, The Riemann problem and a high-resolution Godunov method for a model of compressible two-phase flow. J. Comput. Phys. 212 (2006) 490-526.

[40] A.R. Simpson, Large water hammer pressures due to column separation in sloping pipes. Ph.D. thesis, University of Michigan (1986).

[41] S. Tokareva and E. Toro, HLLC-type Riemann solver for the Baer-Nunziato equations of compressible two-phase flow. J. Comput. Phys. 229 (2010) 3573-3604. 